Teorema de Borsuk-Ulam para formas espaciais esféricas

\author{
Marjory Del Vecchio dos Santos
}





\title{
Teorema de Borsuk-Ulam para formas espaciais esféricas
}

\author{
Marjory Del Vecchio dos Santos
}

Orientador: Prof. Dr. Mauro Flávio Spreafico Coorientador: Prof. Dr. Edivaldo Lopes dos Santos

Tese apresentada ao Instituto de Ciências Matemáticas e de Computação - ICMC-USP, como parte dos requisitos para obtenção do título de Doutor em Ciências Matemática . VERSÃO REVISADA 
Ficha catalográfica elaborada pela Biblioteca Prof. Achille Bassi e Seção Técnica de Informática, ICMC/USP, com os dados fornecidos pelo(a) autor(a)

S237t

Santos, Marjory Del Vecchio dos

Teorema de Borsuk-Ulam para formas espaciais esféricas / Marjory Del Vecchio dos Santos; orientador Mauro Flávio Spreafico; co-orientador Edivaldo Lopes dos Santos. -- São Carlos, 2014. $55 \mathrm{p}$.

Tese (Doutorado - Programa de Pós-Graduação em Matemática) -- Instituto de Ciências Matemáticas e de Computação, Universidade de São Paulo, 2014.

1. Espaços de forma esférica. 2. Teorema de Borsuk-Ulam. 3. Classes de Chern. 4. Grupos periódicos finitos. 5. Cohomologia de grupos. I. Spreafico, Mauro Flávio, orient. II. Santos, Edivaldo Lopes dos, co-orient. III. Título. 
"Once you eliminate the impossible, whatever remains, no matter how improbable, must be the truth."

Sir Arthur Conan Doyle 

Aos meus pais,

Natal e Inez, dedico. 



\section{Agradecimentos}

Agradeço, primeiramente a Deus, que sem Ele, nada seria e nem existiria.

Ao Prof. Dr. Mauro Flávio Spreafico pela orientação.

Ao Prof. Dr. Edivaldo Lopes dos Santos, sem ele não teria concluído esse trabalho, pela disponibilidade em sempre ajudar, pelos conhecimentos transmitidos, pela amizade e pelos conselhos tão valiosos.

À Prof ${ }^{\mathrm{a}}$. Dr ${ }^{\mathrm{a}}$. Denise de Mattos pelo carinho e incentivo.

Ao Prof. Dr. Ozíride Manzoli Neto pelas instruções e apoio.

Aos meus pais, que sempre me incentivaram e me apoiaram nos meus estudos, e pelos sacrifícios que fizeram para que eu estudasse e tivesse uma boa formação.

A minha tia Vera pelas orações.

Ao meu irmão Tiago e minha cunhada Marcela pela amizade, apoio e carinho.

As minhas amigas Ana Paula, Amanda, Lígia e Taciana, agradeço pela amizade e por sempre estarem presentes tanto nos momentos alegres quanto nos momentos difíceis.

Aos meus companheiros de pós-graduação: Matheus, Amanda, Juliana, Northon e demais.

Ao $\mathrm{CNPq}$ pelo apoio financeiro.

A todos que diretamente ou indiretamente contribuíram para que esse sonho se tornasse realidade! 



\section{Resumo}

O objetivo principal deste trabalho é apresentar um estudo sobre o Teorema de Borsuk-Ulam para forma espacial esférica homotópica.

Em nosso trabalho consideramos $X$ uma $n$-forma espacial esférica homotópica a qual admite uma ação livre de $\mathbb{Z}_{p}$, com $p>2$ primo e $f: X \rightarrow \mathbb{R}^{k}$ uma função contínua e, mostramos que sob determinada relação entre os números $n$ e $k$, o conjunto $A(f)$ dos pontos de coincidência de $f$ é não vazio.

Palavras-chave: Borsuk-Ulam, subgrupos de índice primo, formas espaciais esféricas, classes de Chern, cohomologia de grupos finitos, cohomologia periódica, G-coincidências, sequência espectral, fibrações. de aplicações. 



\section{Abstract}

The main objective of this work is to present a study about the BorsukUlam Theorem for homotopic spherical space.

In our work we consider $X$ be a $n$-dimensional homotopic spherical space form which admits a free action of $\mathbb{Z}_{p}$, with $p>2$ prime and $f: X \rightarrow \mathbb{R}^{k}$ be a continuous map and we show that, under certain relations between the numbers $n$ and $k$, the set $A(f)$ is not empty.

Keywords: Borsuk-Ulam, subgroups of prime index, spherical space form, Chern class, cohomology of finite groups, periodic cohomology, $G$-coincidence of maps, spectral sequence, fibration. 



\section{Sumário}

Introdução i

1 Preliminares 1

1.1 Forma espacial esférica . . . . . . . . . . . . . . . . . . . 1

1.2 Espaços de Recobrimento . . . . . . . . . . . . . . . . . . . 2

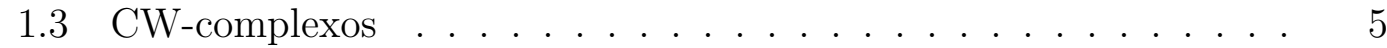

1.4 Resoluções Projetivas . . . . . . . . . . . . . . . . . . . . . . 6

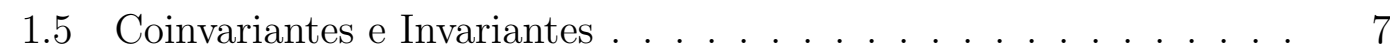

$1.6 \quad$ A (Co)homologia de um Grupo $G \ldots \ldots \ldots$

1.7 Produto Cup . . . . . . . . . . . . . . . . . . . . . . . 11

1.8 Fibrados . . . . . . . . . . . . . . . . . . . . . . . . . . . . . 12

$1.9 \quad$ G-Fibrados principais $\ldots \ldots \ldots \ldots \ldots \ldots$

1.10 Espaços Classificantes . . . . . . . . . . . . . . . . . . . 14

1.11 Fibrações $\ldots \ldots \ldots \ldots \ldots \ldots$

1.12 Sequência Espectral $\ldots \ldots \ldots$

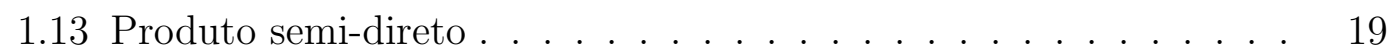

2 Cohomologia de Grupos Finitos 21

2.1 Grupos atuando livremente em esferas . . . . . . . . . . . . . . 21

2.2 Grupos com Cohomologia Periódica . . . . . . . . . . . . . . . . 23

2.3 Anel de Cohomologia de Grupos Periódicos . . . . . . . . . . . . 24

3 Grupos Peródicos Finitos e $\mathbb{Z}_{p}$-ações Livres sobre Formas Espaciais Esféricas 27

$3.1 \quad \mathbb{Z}_{p}$-ações Livres sobre Formas Espaciais Esféricas . . . . . . . . . . 27

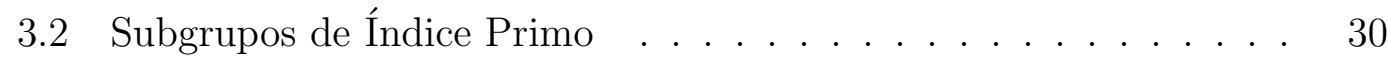


4 Classes de Chern 33

4.1 As Classes de Chern . . . . . . . . . . . . . . . . . . . . . . . 33

5 O Teorema de Borsuk-Ulam para formas espaciais esféricas 39

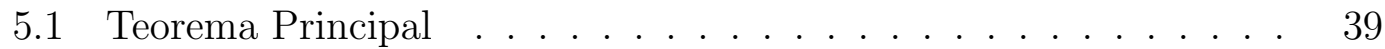

\begin{tabular}{|l|l|}
\hline A Aspectos geométricos do Teorema de Borsuk-Ulam & 43
\end{tabular}

A.1 O teorema de Borsuk-Ulam para $\mathbb{Z}_{p}$-ações livres . . . . . . . . . . 43

\begin{tabular}{ll}
\hline Referências Bibliográficas & 49
\end{tabular}

$\begin{array}{ll}\text { Índice Remissivo } & 55\end{array}$ 


\section{Introdução}

O teorema clássico de Borsuk-Ulam [3], afirma que toda função contínua $f$ da $n$-esfera $S^{n}$ no $n$-espaço euclidiano $\mathbb{R}^{n}$, colapsa pelo menos um par de pontos antípodas, ou seja, existe um ponto $x \in S^{n}$ tal que $f(x)=f(A(x))$, onde $A: S^{n} \rightarrow S^{n}$ denota a aplicação antipodal $A(x)=-x$, para todo $x \in S^{n}$. Esse teorema é uma útil ferramenta da topologia algébrica que tem sido extensamente usado em diferentes áreas. Uma das razões é que existem inúmeras versões e muitas demonstrações conhecidas de cada versão. As técnicas das demonstrações são completamente variadas: os métodos geométricos e elementares, as técnicas algébricas, combinatoriais, a topologia algébrica e muitas outras ferramentas têm sido usadas para prová-lo. O resultado foi primeiramente conjecturado por S. Ulam e provado por Karol Borsuk, em 1933. Desde então, têm sido publicadas diferentes demonstrações, generalizações e aplicações deste famoso teorema (ver, por exemplo, [16]).

Dentre as generalizações bem conhecidas, destacamos pela sua importância, o trabalho de Conner e Floyd [5]. A famosa versão do Teorema de Borsuk-Ulam provada por eles na década de 60, substitui o $n$-espaço Euclidiano $\mathbb{R}^{n}$ por uma $k$-dimensional variedade diferenciável $M^{k}$, para $n \geq k$, com a hipótese adicional que a induzida da $f$ no nível 
$n$ se anula na $\mathbb{Z}_{2}$-cohomologia. Em [2], Biasi, De Mattos e Dos Santos estenderam o resultado de Conner e Floyd substituindo a $k$-variedade diferenciável por uma $k$-variedade generalizada, provando o seguinte

Teorema [2, Teorema 1.1]. Sejam $f: S^{n} \rightarrow M^{k}$ uma função contínua da n-esfera $S^{n}$ em uma variedade generalizada $M^{k}$ e $A(f)=$ $\{x \in X ; f(x)=f(-x)\}$.

i) se $n>k$, então $\operatorname{dim} A(f) \geq n-k$;

ii) se $n=k$ e $f^{*}: H^{n}\left(M^{n}, \mathbb{Z}_{2}\right) \rightarrow H^{n}\left(S^{n}, \mathbb{Z}_{2}\right)$ for nula, então $A(f) \neq$ $\emptyset$.

Existem muitas formulações do Teorema de Borsuk-Ulam. Consideremos a seguinte formulação geral do teorema de Borsuk-Ulam:

Sejam $X, Y$ espaços topológicos, $G$ um grupo finito agindo livremente sobre $X$ e $f: X \rightarrow Y$ uma aplicação contínua. Dizemos que um ponto $x \in X$ é um ponto de $G$-coincidência de $f$ se a função $f$ leva a órbita $G x$ em um único ponto. O conjunto de todos os pontos de $G$-coincidência de $f$ é denotado por $A(f)$.

Na formulação geral acima de $G$-coincidência quando o domínio $X$ é um espaço satisfazendo determinadas condições e contradomínio $Y$ é um $C W$ - complexo, o problema foi abordado em vários artigos, a saber, [7, 8, 9, 10], [13, 14] e [21].

No caso em que o domínio é a $n$-esfera ou uma esfera de homologia e o contradomínio $Y$ é o espaço euclidiano $\mathbb{R}^{k}$, o problema foi também abordado em vários artigos (ver por exemplo [21]).

Recentemente, D. L. Gonçalves, O. Manzoli Neto e M. Spreafico em [1], provaram o Teorema de Borsuk-Ulam para espaços de forma esférica que admitem ações livres do grupo cíclico $G=\mathbb{Z}_{2}$. Mais especificamente, eles provaram o seguinte resultado. 
Teorema [11, Teorema 1.4]. Seja $X=X(2 n-1)$ um espaço de forma esférica $(2 n-1)$-dimensional, e $\tau$ uma involução livre em $X$, com $n>2$. Então o Teorema de Borsuk-Ulam é válido para a tripla $\left(X(2 n-1), \tau ; R^{2 n-1}\right)$ se, e somente se, $\pi_{1}(X)$ não possui elementos de ordem 2.

O objetivo do nosso trabalho foi obter uma versão do teorema de Borsuk-Ulam considerando o domínio $X$ no problema geral acima como sendo as $n$-formas espaciais esféricas homotópicas e $G$ o grupo cíclico $\mathbb{Z}_{p}$, com $p>2$ primo.

A seguir, apresentamos o principal resultado deste trabalho.

Teorema (5.1.1). Dada uma forma espacial esférica homotópica (2n-1)-dimensional $X$ com uma ação livre de $\mathbb{Z}_{p}, p>2$ primo, e uma aplicação contínua $f: X \rightarrow \mathbb{R}^{m}$ com $2 n-1 \geq m(p-1)$, então o conjunto de coincidências $A(f) \neq \emptyset$. Mais ainda,

$$
\operatorname{dim} A(f) \geq \operatorname{dim} X-m(p-1)
$$

No desenvolvimento da tese usamos conceitos de topologia e álgebra, tais como classes de Chern, sequências espectrais, cohomologia de grupos, grupos periódicos finitos, grupos atuando em esferas, espaços de forma esférica, entre outros.

A seguir relatamos sucintamente o objeto de estudo de cada capítulo.

No capítulo 1 encontram-se definições e propriedades básicas relativas ao desenvolvimento do projeto tais como espaço de forma esférica, cohomologia de grupos, fibrações, sequências spectrais, etc. A intenção deste capítulo é facilitar a leitura e entendimento dos capítulos seguintes.

No capítulo 2 encontram-se definições e resultados sobre 
cohomologia de grupos finitos, especificamente sobre cohomologia de grupos periódicos finitos, os quais são geradores de espaços de forma esférica. O conhecimento do anel de cohomologia módulo $p$ de tais grupos foi de fundamental importância para o desenvolvimento do nosso trabalho.

No capítulo 3 nós apresentamos uma caracterização das formas espaciais esféricas que admitem $\mathbb{Z}_{p}$-ações livres (ver Teorema 3.1.1). A existência de tais $\mathbb{Z}_{p}$-espaços livres está diretamente relacionada com os subgrupos de índice primo dos grupos periódicos finitos. $\mathrm{Na}$ seção 3.2, asseguramos a existência de tais subgrupos, e desta forma, a existência de espaços de forma esférica que admitem uma ação livre de $\mathbb{Z}_{p}$.

No capítulo 4 encontra-se um estudo sobre classes de Chern no que diz respeito a sua relação com o conjunto de coincidências $A(f)$.

No capítulo 5, usando as ferramentas desenvolvidas nos capítulos anteriores, demonstramos nosso resultado principal, um teorema do tipo Borsuk-Ulam no caso em que o domínio é uma forma espacial esférica homotópica. Demostramos que o conjunto de coincidências é não vazio e também damos uma estimativa para sua dimensão.

No apêndice A apresentamos alguns aspectos geométricos do Teorema de Borsuk-Ulam para o caso de ações livres do grupo cíclico $\mathbb{Z}_{p}$, apresentando uma versão equivalente deste resultado em termos de aplicações $\mathbb{Z}_{p}$-equivariantes. 


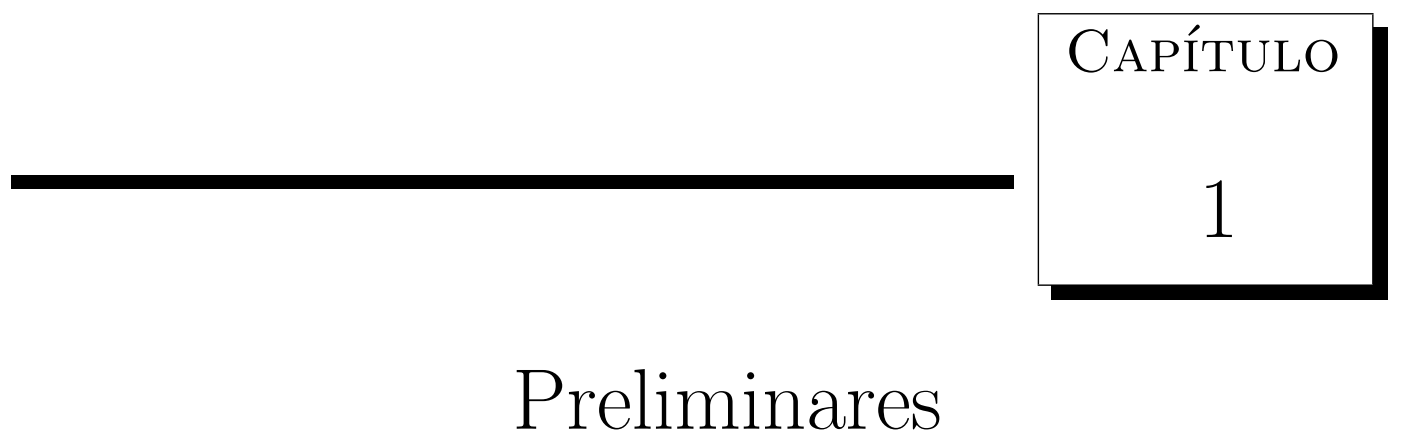

Neste capítulo apresentamos algumas definições e resultados que serão de bastante utilidade para o desenvolvimento dos capítulos posteriores. O objetivo desse capítulo é facilitar a leitura e entendimento dos próximos capítulos e sendo assim, será omitida a maioria das demonstrações dos resultados apresentados. Suas principais referências são [1], 19], 20], 22]

\subsection{Forma espacial esférica}

Definição 1.1.1 Uma n-forma espacial esférica é uma variedade Riemanniana conexa completa de curvatura constante positiva, ou seja, é exatamente o quociente $S^{n} / \Gamma$ da esfera pela ação de um subgrupo $\Gamma \subset O(n+1, \mathbb{R})$ de isometrias livres de ponto fixo. Uma classificação completa das formas espaciais esféricas foi feita por J. Wolf em [22].

Desde que $S^{n} / \Gamma$ é conexa, $H_{0}\left(S^{n} / \Gamma ; \mathbb{Z}\right) \simeq \mathbb{Z}$. Como $S^{n}$ e $p: S^{n} \rightarrow S^{n} / \Gamma$ é um recobrimento, então $S^{n} / \Gamma$ é compacta e $H_{n}\left(S^{n} / \Gamma ; \mathbb{Z}\right)$ é isomorfo a $\mathbb{Z}$ ou 
$\mathbb{Z}_{2}$, dependendo se é orientável ou não, respectivamente. Se $n$ é par, então as únicas formas espaciais esféricas (a menos de isometria) são a esfera e o espaço projetivo. Se $n$ é impar, então $S^{n} / \Gamma$ é orientável pois as representações do grupo $\Gamma$ em $O(n+1, \mathbb{R})$ originam-se de representações complexas e portanto se reduzem a $S O(n+1)$. Também o grupo das transformações deck é $\Gamma$. Usando a sequência exata de homotopia de $p$ segue que $\pi_{1}\left(S^{n} / \Gamma\right) \simeq \Gamma$ e $\pi_{k}\left(S^{n} / \Gamma\right) \simeq \pi_{k}\left(S^{n}\right)$, para $k>1$.

Definição 1.1.2 Seja $\Sigma^{m}$ um $C W$-complexo m-dimensional com o mesmo tipo de homotopia da m-esfera. Dada uma ação celular livre $\gamma$ de um grupo finito $G$ em $\Sigma^{m}$, denotamos por $\Sigma^{m} / \gamma(G)$ o espaço de órbitas correspondente, chamado espaço de forma esférica homotópica ou forma espacial esférica homotópica mdimensional.

\subsection{Espaços de Recobrimento}

Para definirmos espaços de recobrimento assumimos que os espaços topológicos utilizados são conexos por caminhos e localmente conexos por caminhos (e portanto, conexos). Os resultados desta seção podem ser encontrados em [19].

Definição 1.2.1 Sejam $X$ e $Y$ dois G-espaços e $f: X \longrightarrow Y$ uma aplicação contínua. Se $f(g x)=g f(x)$ para todo $x \in X$ e todo $g \in G$, então $f$ é chamada de aplicação equivariante.

Definição 1.2.2 Seja $X$ um espaço topológico. Um espaço de recobrimento de $X$ é um par $(\widetilde{X}, p)$, com $\widetilde{X}$ um espaço de recobrimento e $p: \widetilde{X} \rightarrow X$ uma aplicação contínua tal que:

(1) $p$ é sobrejetora.

(2) Todo ponto $x \in X$ possui uma vizinhança $U$ aberta, conexa por caminhos de modo que a restrição de $p$ a cada componente conexa $\widetilde{U}$ de $p^{-1}(U)$ é um homeomorfismo. 
Observação 1.2.1 Denominamos a aplicação $p$ e a vizinhança $U$ respectivamente de projeção de recobrimento $e$ vizinhança elementar. Ainda, o conjunto $p^{-1}(x)$ é denominado fibra no ponto $x \in X$.

Exemplo 1.2.1 Seja $p: \mathbb{R} \rightarrow S^{1}$ dada por $p(t)=($ sent, cost $), t \in \mathbb{R}$. Então $(\mathbb{R}, p)$ é um recobrimento de $S^{1}$. Além disso, todo subintervalo aberto de $S^{1}$ pode ser visto como uma vizinhança elementar.

Observação 1.2.2 Sejam $(\tilde{X}, p)$ e $(\tilde{Y}, q)$ recobrimentos de $X$ e $Y$ respectivamente. Então $(\widetilde{X} \times \tilde{Y}, p \times q)$ é recobrimento de $X \times Y$, sendo a aplicação $p \times q$ definida como $(p \times q)(x, y)=(p(x), q(y))$. Agora, se $U$ e $V$ são vizinhanças elementares de $x \in X$ e $y \in Y$ então $U \times V$ é uma vizinhança elementar de $(x, y) \in X \times Y$.

Proposição 1.2.1 Sejam $(\tilde{X}, p)$ um recobrimento de $X, \widetilde{x_{0}} \in \widetilde{X}$ e $x_{0}=$ $p\left(\widetilde{x_{0}}\right)$. Então o homomorfismo induzido $p_{*}: \pi_{1}\left(\widetilde{X}, \widetilde{x_{0}}\right) \longrightarrow \pi_{1}\left(X, x_{0}\right)$ é um monomorfismo.

Definição 1.2.3 Seja $(\widetilde{X}, p)$ um recobrimento de $X$. Um homeomorfismo $\varphi$ : $\widetilde{X} \rightarrow \widetilde{X}$ é dito transformação de recobrimento (ou Deck transformação) se $p \circ \varphi=p$. Ainda, o conjunto de todas as transformações de recobrimento (denotado por $A(\widetilde{X}, p)$ ) é um grupo em relação à composição.

Proposição 1.2.2 O grupo $A(\tilde{X}, p)$ atua livremente sobre $\tilde{X}$, isto é, g. $\widetilde{x}=\widetilde{x}$ se, e somente se, $g=1$.

Definição 1.2.4 Um recobrimento $(\widetilde{X}, p)$ de $X$ é dito recobrimento universal de $X$ se $\widetilde{X}$ é simplesmente conexo, isto é, se $\widetilde{X}$ é conexo por caminhos $e$ $\pi_{1}(\widetilde{X}, \widetilde{x})=0$, para todo $\widetilde{x} \in \widetilde{X}$. 
Definição 1.2.5 Um recobrimento $(\widetilde{X}, p)$ é dito recobrimento regular se $p_{*}\left(\pi_{1}(\widetilde{X}, \widetilde{x})\right)$ é um subgrupo normal de $\pi_{1}(X, x)$. Ainda, esta condição independe da escolha do ponto base $\widetilde{x} \in p^{-1}(x)$.

Exemplo 1.2.2 Todo recobrimento universal é regular.

Definição 1.2.6 Se $(\widetilde{X}, p)$ é um espaço de recobrimento de $X$, o número cardinal comum dos conjuntos $p^{-1}(x), x \in X$, é chamado de número de folhas do recobrimento. Nós dizemos que o recobrimento é de $n$-folhas se $\# p^{-1}(x)=n$ e de infinitas folhas se $\# p^{-1}(x)=\infty$.

Proposição 1.2.3 Seja $(\tilde{X}, p)$ um recobrimento regular de $X$. Então $A(\tilde{X}, p)$ é isomorfo ao grupo quociente $\pi_{1}(X, x) / p_{*}\left(\pi_{1}(\tilde{X}, \widetilde{x})\right)$, para todo $x \in X$ e todo $\widetilde{x} \in p^{-1}(x)$.

Demonstração. [19].

Proposição 1.2.4 Seja $(\tilde{X}, p)$ um recobrimento regular de $X$. O grupo $A(\widetilde{X}, p)$ atua transitivamente sobre $p^{-1}(x)$ se, e somente se, $(\widetilde{X}, p)$ é um recobrimento regular de $X$.

Definição 1.2.7 Um grupo $G$ de homeomorfismos de $X$ é dito propriamente descontínuo se todo ponto $x \in X$ possui uma vizinhança $V$ tal que, para todo $g \in G, g \neq 1$, tem-se $g . V \cap V=\emptyset$.

Proposição 1.2.5 Seja $Y$ um espaço conexo e localmente conexo por caminhos. Seja G um grupo de homeomorfismos de Y propriamente descontinua. Considere $p: Y \rightarrow Y / G$ a projeção natural de $Y$ no espaço quociente $Y / G$. Então $(Y, p) e ́$ um recobrimento regular de $Y / G$ e $G=A(Y, p)$.

Proposição 1.2.6 Seja $(\tilde{X}, p)$ um recobrimento universal de $X$. Então $A(\tilde{X}, p) \simeq$ $\pi_{1}(X)$ e a ordem de $\pi_{1}(X)$ é igual ao número de folhas do recobrimento $(\tilde{X}, p)$.

Observação 1.2.3 $A(\tilde{X}, p)=\{\phi: \tilde{X} \rightarrow \tilde{X} \mid \phi$ é automorfismo $\}$ 


\subsection{CW-complexos}

Definição 1.3.1 Dado um espaço $X$ de Hausdorff, dizemos que $X$ admite uma estrutura de $\mathbf{C W}$-complexo se possui uma coleção de subconjuntos fechados $\sigma_{j}^{q}$ (onde $q$ representa dimensão $(q=0,1,2, \ldots)$ e $j$ varia sobre um conjunto de indices $J_{q}$ ), e uma família de subespaços fechados $X^{0} \subset X^{1} \subset \ldots \subset X^{q} \subset \ldots$ com $X^{q}=\bigcup_{\substack{p \leq q \\ j \in J_{p}}} \sigma_{j}^{p}$ (por definiçãa $\left.X^{-1}=\emptyset\right)$, e fronteira dada por $f_{j}^{q}=\sigma_{j}^{q} \cap X^{q-1}$, satisfazendo as seguintes propriedades:

(i) $\sigma_{i}^{p}-f_{i}^{p}$ intercepta $\sigma_{j}^{q}-f_{j}^{q}$ somente quando $p=q$ e $i=j$.

(ii) $X=\bigcup_{q} X^{q}$.

(iii) Para cada $\sigma_{j}^{q}$, existe uma aplicação característica $\phi_{j}^{q}: D^{q} \rightarrow \sigma_{j}^{q}$ (onde $D^{q}$ é o disco de dimensão q) que leva $S^{q-1}$ (esfera de dimensão $q-1$ ) sobre $f_{j}^{q}$, e aplica $D^{q}-S^{q-1}$ homeomorficamente sobre $\sigma_{j}^{q}-f_{j}^{q}\left(S^{-1}\right.$ é o conjunto vazio).

(iv) $f_{j}^{q}$ intercepta um número finito de conjuntos $\left(\sigma_{i}^{q}-f_{i}^{q}\right), i \in J_{q}$.

(v) Um subconjunto $Y$ de $X$ é fechado se $Y \cap \sigma_{j}^{q}$ é fechado em $\sigma_{j}^{q}, \forall q$ e $\forall j \in J_{q}$, onde $\sigma_{j}^{q}$ possui a topologia quociente de $D^{q}\left(\right.$ via $\left.\phi_{j}^{q}\right)$.

Notação: Em um $C W$-complexo $X, e^{q}$ denotará a célula aberta de dimensão $q$ :

$$
e^{q}=\sigma^{q}-f^{q} .
$$

Exemplo 1.3.1 Seja $X=\mathbb{R}$. Podemos dar a $\mathbb{R}$ uma estrutura natural de $C W$ complexo, onde as 0 -células e 1-células são dadas, respectivamente, por $e_{n}^{0}=\{n\}$ $e e_{n}^{1}=(n, n+1), n \in \mathbb{Z}$.

Exemplo 1.3.2 Seja $X=S^{n}$ (n-esfera). Uma estrutura de $C W$-complexo sobre $S^{n}$ pode ser dada por uma 0-célula e uma $n$-célula, ou seja, $S^{n}=e^{0} \cup e^{n}$.

Definição 1.3.2 Um G-complexo é um $C W$-complexo $X$ munido de uma ação de $G$ em $X$ que permuta as células, isto é, se $S$ representa o conjunto das células de $X$, então $g S=S$, para todo $g$ em $G$. Se a ação de $G$ em $X$ permuta livremente as células, dizemos que $X$ é um G-complexo livre. 
Observação 1.3.1 É importante notar que, pelo fato da ação de $G$ em X induzir um homomorfismo dado por

$$
\begin{aligned}
\varphi: G & \rightarrow \text { Homeo }(X) \\
g & \mapsto \varphi_{g}: X \rightarrow X
\end{aligned}
$$

tal que $\varphi_{g}(x)=g \cdot x$, temos que, se $\sigma$ é uma célula de $X$, então $g \cdot \sigma$ também é uma célula de $X$ cuja dimensão é a mesma de $\sigma$ (ou seja, $\varphi_{g}$ preserva dimensão).

Teorema 1.3.1 Se $X$ é um G-complexo livre contrátil, então o complexo de cadeia celular aumentado de $X$ :

$$
\cdots \longrightarrow C_{n}(X) \stackrel{\partial_{n}}{\longrightarrow} C_{n-1}(X) \longrightarrow \cdots \longrightarrow C_{1}(X) \stackrel{\partial_{1}}{\longrightarrow} C_{0}(X) \stackrel{\varepsilon}{\longrightarrow} \mathbb{Z} \longrightarrow 0
$$

é uma resolução livre de $\mathbb{Z}$ sobre $\mathbb{Z} G$.

Demonstração. [4]

\subsection{Resoluções Projetivas}

Definição 1.4.1 Considere $R$ um anel com unidade e $M$ um $R$-módulo à esquerda. Uma resolução de $M$ sobre $R$, ou uma $R$-resolução de $M$, é uma sequência exata de $R$-módulos

$$
C: \ldots \longrightarrow C_{n+1} \stackrel{\partial_{n+1}}{\longrightarrow} C_{n} \stackrel{\partial_{n}}{\longrightarrow} C_{n-1} \stackrel{\partial_{n-1}}{\longrightarrow} \cdots
$$

a qual satisfaz as seguintes condições:

(R1) $C_{-1}=M$

(R2) $C_{n}=0, \forall n<-1$

Equivalentemente, podemos escrever esta definição da seguinte forma: uma resolução de $M$ sobre $R$, ou uma $R$-resolução de $M$, é uma sequência exata de $R$-módulos

$$
C: \ldots \longrightarrow C_{2} \stackrel{\partial_{2}}{\longrightarrow} C_{1} \stackrel{\partial_{1}}{\longrightarrow} C_{0} \stackrel{\varepsilon}{\longrightarrow} M \longrightarrow 0 .
$$


Definição 1.4.2 A aplicação $\epsilon: C_{0} \rightarrow M$ é chamada aplicação aumentação. Se cada $C_{i}$ é um $R$-módulo livre, dizemos que a resolução é livre. Se cada $C_{i}$ é um $R$-módulo projetivo, dizemos que a resolução é projetiva.

Notação: $\epsilon: C \rightarrow M$ denotará uma resolução de $M$.

Proposição 1.4.1 [4, I.8.2] São equivalentes as seguintes condições para um $R$-módulo P:

(i) P é projetivo.

(ii) Toda seqüência exata $0 \longrightarrow M^{\prime} \longrightarrow M \longrightarrow P \longrightarrow 0$ cinde.

(iii) P é somando direto de um módulo livre.

Proposição 1.4.2 Dado um $R$-módulo $M$ sempre existe uma $R$-resolução livre de $M$.

Demonstração. [19]

\subsection{Coinvariantes e Invariantes}

Sejam $G$ um grupo e $M$ um $R G$-módulo (à esquerda).

Definição 1.5.1 O grupo dos coinvariantes de $M$, o qual denotamos por $M_{G}$, é dado por $M_{G}=M / A$, onde $A$ é o subgrupo aditivo dado por $A=<g \cdot m-m ; g \in$ Ge $m \in M>$.

Observação 1.5.1 O nome coinvariantes vem do fato de $M_{G}$ ser o maior quociente de $M$ no qual $G$ atua trivialmente.

Proposição 1.5.1 [4, II.2.1.] $M_{G} \simeq R \otimes_{R G} M$, onde $R$ é visto como um $R G$ módulo (à direita) com $G$-ação trivial.

Definição 1.5.2 Seja $M$ um RG-módulo (à esquerda). $O$ grupo dos invariantes de $M$, denotado por $M^{G}$, é dado por:

$$
M^{G}=\{m \in M ; g \cdot m=m, \forall g \in G\} .
$$


Proposição 1.5.2 $\operatorname{Hom}_{R G}(R, M) \simeq M^{G}$, onde $R$ é um $R G$-módulo com $G$-ação trivial.

Demonstração. Definindo $\psi: \operatorname{Hom}_{R G}(R, M) \rightarrow M^{G}$ por $\psi(f):=f(1)$, temos que $\psi$ é um isomorfismo.

Observação 1.5.2 Todo RG-módulo (à esquerda) $M$ pode ser considerado como um RG-módulo (à direita), definindo a seguinte $G$-ação em $M$ :

$$
\begin{aligned}
\varphi: M \times G & \rightarrow \quad M \\
(m, g) & \mapsto m * g=g^{-1} \cdot m, \quad \forall g \in G, \forall m \in M .
\end{aligned}
$$

\subsection{A (Co)homologia de um Grupo G}

Seja $R$ um anel comutativo com unidade e considere o anel grupo $R G$.

Antes de definirmos (co)homologia, vamos considerar alguns resultados importantes sobre $\otimes_{R G}$ e $\mathrm{Hom}_{R G}$.

Definição 1.6.1 Sejam $M$ e $N$ RG-módulos. Então, $M$ e $N$ são naturalmente R-módulos. A G-ação diagonal, definida em $M \otimes_{R} N$, é dada por:

$$
g \cdot(m \otimes n)=g \cdot m \otimes g \cdot n
$$

Proposição 1.6.1 Sejam $M$ e N RG-módulos (à esquerda). Temos

$$
M \otimes_{R G} N=\left(M \otimes_{R} N\right)_{G}:=\frac{M \otimes_{R} N}{A}
$$

onde $A=<g \cdot m \otimes g \cdot n-m \otimes n ; \forall m \otimes n \in M \otimes_{R} N, \forall g \in G>$.

Demonstração. Ver [19]

Corolário 1.6.1 $M \otimes_{R G} N \simeq N \otimes_{R G} M$.

Demonstração. $\quad M \otimes_{R G} N \simeq\left(M \otimes_{R} N\right)_{G} \simeq\left(N \otimes_{R} M\right)_{G}=N \otimes_{R G} M$. 
Sejam $M$ e $N R G$-módulos (à esquerda), e consideremos $\operatorname{Hom}_{R}(M, N)$.

A ação de $G$ em $M$ e $N$ induz uma ação de $G$ em $H_{o m}(M, N)$, dada por

$$
\begin{array}{ccc}
G \times \operatorname{Hom}_{R}(M, N) & \rightarrow & \operatorname{Hom}_{R}(M, N) \\
(g, f) & \mapsto & g \cdot f
\end{array}
$$

tal que $g \cdot f(x)=g f\left(g^{-1} \cdot x\right) ; g \in G, f \in H_{R}(M, N)$ e $x \in M$.

Observação 1.6.1 O uso de $g^{-1}$ para definir a ação é necessário devido à contravariância de Hom na primeira variável. Compensamos esta contravariância, convertendo $M$ a um $R G$-módulo à direita, considerando $m * g=$ $g^{-1} \cdot m$.

Deste modo, a ação fica:

$$
g \cdot f(m)=g f\left(g^{-1} \cdot m\right)=g f(m * g) .
$$

Assim, $\operatorname{Hom}_{R}(M, N)$ será um RG-módulo (à esquerda).

Proposição 1.6.2 $\operatorname{Hom}_{R G}(M, N)=\operatorname{Hom}_{R}(M, N)^{G}$.

Demonstração. Ver [19]

Veremos, agora, a definição de (co)homologia de um grupo $G$, considerando o caso $R=\mathbb{Z}$.

Definição 1.6.2 Sejam

$$
\cdots \longrightarrow F_{n} \stackrel{\partial_{n}}{\longrightarrow} F_{n-1} \longrightarrow \cdots \longrightarrow F_{1} \stackrel{\partial_{1}}{\longrightarrow} F_{0} \stackrel{\varepsilon}{\longrightarrow} \mathbb{Z} \longrightarrow 0
$$

uma resolução projetiva de $\mathbb{Z}$ sobre $\mathbb{Z} G$ e $M$ um $\mathbb{Z} G$-módulo (à esquerda).

Podemos formar os complexos de cadeia e cocadeia, respectivamente:

$F \otimes_{Z G} M: \cdots \longrightarrow F_{n} \otimes_{Z G} M \stackrel{\bar{\partial}_{n}}{\longrightarrow} F_{n-1} \otimes_{Z G} M \longrightarrow \cdots \longrightarrow F_{1} \otimes_{Z G} M \stackrel{\bar{\partial}_{1}}{\longrightarrow} F_{0} \otimes_{Z G} M \longrightarrow 0$ 
$\operatorname{Hom}_{Z G}(F, M): 0 \longrightarrow \operatorname{Hom}_{Z G}\left(F_{0}, M\right) \stackrel{\delta^{0}}{\longrightarrow} \operatorname{Hom}_{Z G}\left(F_{1}, M\right) \stackrel{\delta^{1}}{\longrightarrow} \cdots \longrightarrow \operatorname{Hom}_{Z G}\left(F_{n}, M\right) \longrightarrow \cdots$

$O$ operador bordo é dado por $\bar{\partial}_{n}:=\partial_{n} \otimes i d e$, o operador cobordo, por

$$
\begin{array}{cl}
\delta^{n}: \operatorname{Hom}_{Z G}\left(F_{n}, M\right) & \rightarrow \operatorname{Hom}_{Z G}\left(F_{n+1}, M\right) \\
f & \mapsto \delta^{n}(f):=f \circ \partial_{n+1}
\end{array}
$$

(a) O n-ésimo grupo de homologia de $G$ com coeficientes em $M$ é definido por

$$
H_{n}(G, M):=H_{n}\left(F \otimes_{Z G} M\right)
$$

(b) O n-ésimo grupo de cohomologia de $G$ com coeficientes em $M$ é definido por

$$
H^{n}(G, M):=H^{n}\left(\operatorname{Hom}_{Z G}(F, M)\right) .
$$

Observação 1.6.2 Tomando $M=\mathbb{Z}$, com $G$-ação trivial, temos

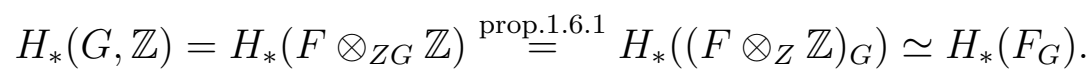

Proposição 1.6.3 Dado um $\mathbb{Z} G$-módulo $M$, temos os isomorfismos:

$$
\begin{aligned}
& H_{0}(G, M) \simeq M_{G} . \\
& H^{0}(G, M) \simeq M^{G} .
\end{aligned}
$$

Demonstração. ver 4]

Proposição 1.6.4 (Interpretação topológica da (co)homologia de um grupo) Sejam $G$ um grupo, $Y$ um $K(G, 1)$-complexo e $M$ um $\mathbb{Z} G$-módulo. Então

$$
H_{*}(G, M) \simeq H_{*}(Y, \mathcal{M})
$$




$$
H^{*}(G, M) \simeq H^{*}(Y, \mathcal{M})
$$

onde $\mathcal{M}$ é um sistema de coeficientes locais em $Y$ associado ao $\mathbb{Z} G$-módulo $M$. Em particular se a G-ação em $M$ é trivial então $\mathcal{M}=M$.

Demonstração. ver [19]

Exemplo 1.6.1 Temos que $S^{1}$ é um $K(\mathbb{Z}, 1)$-complexo. Logo

$$
H_{i}(\mathbb{Z}) \simeq H_{i}\left(S^{1}\right) \simeq H^{i}\left(S^{1}\right) \simeq H^{i}(\mathbb{Z})=\left\{\begin{array}{l}
\mathbb{Z} \text { se } i=0,1 \\
0, \text { se } i \neq 0,1
\end{array}\right.
$$

Exemplo 1.6.2 Temos que $T^{2}$ é um $K(\mathbb{Z} \oplus \mathbb{Z}, 1)$-complexo. Logo

$$
H_{i}(\mathbb{Z} \oplus \mathbb{Z}) \simeq H_{i}\left(T^{2}\right) \simeq H^{i}\left(T^{2}\right) \simeq H^{i}(\mathbb{Z} \oplus \mathbb{Z})=\left\{\begin{array}{l}
\mathbb{Z} \text { se } i=0,2 ; \\
\mathbb{Z} \oplus \mathbb{Z}, \text { se } i=1 \\
0, \text { se } i \neq 0,1,2 .
\end{array}\right.
$$

\subsection{Produto Cup}

Sejam $\mathrm{M}$ e $\mathrm{N}$ dois $\mathbb{Z} G$-módulos. Se $F \stackrel{\varepsilon}{\rightarrow} \mathbb{Z}$ é uma resolução projetiva de $\mathbb{Z}$ sobre $\mathbb{Z} G$. Então $F \otimes F \stackrel{\varepsilon \otimes \varepsilon}{\longrightarrow} \mathbb{Z}$ é uma resolução projetiva de $\mathbb{Z}$ sobre $\mathbb{Z}(G \times G)($ ver [4]).

Sejam $f \in H_{o m}(F, M)$ e $g \in H_{o m}(F, N)$. Definimos $f \times g \in H_{o m} \times m_{G \times G}(F \otimes$ $F, M \otimes N)$ por $(f \times g)(x \otimes y)=(-1)^{p q} f(x) \otimes g(y) \operatorname{com} x \in F_{p}$ e $y \in F_{q}$.

Sejam G um grupo e $d: G \longrightarrow G \times G$ definida por $d(g)=(g, g)$ (aplicação diagonal)

Definição 1.7.1 A composta

$$
H^{p}(G, M) \otimes H^{q}(G, N) \stackrel{\times}{\longrightarrow} H^{p+q}(G \times G, M \otimes N) \stackrel{d^{*}}{\longrightarrow} H^{p+q}(G, M \otimes N)
$$


é chamado de produto cup e é denotado por $\cup$.

Assim dados $u \in H^{p}(G, M)$ e $v \in H^{q}(G, N)$ temos $u \cup v:=d^{*}(u \times v)$

\subsection{Fibrados}

Definição 1.8.1 Um fibrado é uma quádrupla $\xi=(E, p, B, F)$, onde $E, B$ e $F$ são espaços topológicos e $p: E \rightarrow B$ é uma aplicação contínua e sobrejetora que satisfaz a condição de trivialidade local descrita abaixo.

Existe uma cobertura de $B$ por abertos $U_{\alpha}$, denominados vizinhanças coordenadas, e associado a cada aberto na cobertura temos um homeomorfismo $\phi_{\alpha}: p^{-1}\left(U_{\alpha}\right) \rightarrow U_{\alpha} \times F$, o qual chamamos uma aplicação coordenada, tal que o seguinte diagrama é comutativo:

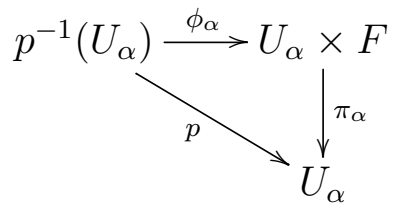

ou seja, $\pi_{\alpha} \circ \phi_{\alpha}=p$, onde $\pi_{\alpha}$ é a projeção sobre $U_{\alpha}$. Assim, localmente o espaço E se parece com um espaço produto. O espaço E é chamado espaço total, $B$ é chamado espaço base e $F$ a fibra. A comutatividade do diagrama anterior significa que $\phi_{\alpha}$ leva cada fibra $p^{-1}(b)$ homeomorficamente sobre a cópia $\{b\} \times F$ de $F$, assim a denominação do espaço $F$ como fibra se justifica.

Definição 1.8.2 A aplicação p é chamada aplicação fibrada ou projeção. O conjunto de todos os pares $\left\{\left(U_{\alpha}, \phi_{\alpha}\right)\right\}_{\alpha}$ de vizinhanças coordenadas e suas aplicações coordenadas associadas é chamado uma trivialização local do fibrado. Geralmente letras gregas como $\xi, \eta, \omega, \cdots$ são usadas para denotar fibrados. Também é comum denotar um fibrado $\xi=(E, p, B, F)$ simplesmente por $p$ : $E \rightarrow B$.

Exemplo 1.8.1 O fibrado produto $\pi: B \times F \rightarrow B$, onde $\pi$ denota a projeção sobre $B$. 
Exemplo 1.8.2 Considere um fibrado $\xi=(E, p, B, F)$ e um subconjunto $A \subset B$, então $\left.p\right|_{p^{-1}(A)}: p^{-1}(A) \rightarrow A$ é um fibrado, chamado fibrado restrição de E sobre $A$, o qual denotamos por $\left.\xi\right|_{A}$.

Exemplo 1.8.3 Considere dois fibrados $\xi_{i}=\left(E_{i}, p_{i}, B_{i}, F_{i}\right), i=1,2$, então $p_{1} \times$ $p_{2}: E_{1} \times E_{2} \rightarrow B_{1} \times B_{2}$ é um fibrado com fibra $F_{1} \times F_{2}$, o qual denotamos por $\xi_{1} \times \xi_{2}$.

Exemplo 1.8.4 Considere dois fibrados $\xi_{i}=\left(E_{i}, p_{i}, B, F_{i}\right), i=1,2$, com mesmo espaço base $B$. A restrição do fibrado $\xi_{1} \times \xi_{2}$ sobre a diagonal $\{(b, b) \in B \times B\}$, a qual é homeomorfa a $B$, é um fibrado chamado soma direta, o qual denotamos por

$$
\xi_{1} \oplus \xi_{2}=\left(E_{1} \oplus E_{2}, p_{1} \oplus p_{2}, B, F_{1} \oplus F_{2}\right)
$$

A soma direta de fibrados é também denominada a soma de Whitney.

Exemplo 1.8.5 Sejam $\xi=(E, p, B, F)$ um fibrado e $f: B^{*} \rightarrow B$ uma aplicação contínua. O fibrado induzido de $\xi$ por $f$, o qual denotamos por $f^{*}(\xi)$, tem como espaço base $B^{*}$ e seu espaço total é definido como sendo o sub-espaço

$$
f^{*}(E)=\left\{\left(b^{*}, x\right) \in B^{*} \times E \mid f\left(b^{*}\right)=p(x)\right\} .
$$

A aplicação fibrada é dada pela projeção $p^{*}: f^{*}(E) \rightarrow B^{*}, p^{*}\left(b^{*}, x\right)=b^{*}$. $O$ fibrado induzido de $\xi$ por $f, f^{*}(\xi)=\left(f^{*}(E), p^{*}, B^{*}, F\right)$ também é chamado o pullback de $\xi$ por $f$.

Definição 1.8.3 Um fibrado $\xi=(E, p, B, F)$ é chamado trivial se $\xi$ é B-isomorfo ao fibrado produto $\pi: B \times F \rightarrow B$.

\section{$1.9 \quad$ G-Fibrados principais}

Definição 1.9.1 Um G-espaço é um espaço de Hausdorff $X$ junto com uma $G$ ação de um grupo topológico $G$ sobre $X$.

Se $X$ é um G-espaço, então o subespaço $G x=[x]=\{g x \in X \mid g \in G\}$ é chamado a órbita do ponto $x$ pela ação de $G$. Denotaremos por $X / G$ o conjunto de todas as órbitas $G x$ pela ação de $G$ sobre $X$. 
Definição 1.9.2 Seja $\pi_{X}: X \rightarrow X / G$ a projeção canônica definida por $x \mapsto$ $G x$. O espaço quociente $X / G$, munido da topologia quociente induzida por $\pi_{X}$, é chamado o espaço de órbitas de $X$ pela ação de $G$. Além disso, o G-espaço $X$ determina um fibrado $\xi_{X}=\left(X, \pi_{X}, X / G\right)$.

Definição 1.9.3 Um fibrado $\xi=(X, p, B)$, sobre um espaço base $B$, é chamado um $G$-fibrado se $\xi$ é isomorfo a $\xi_{X}$, para alguma estrutura de G-espaço sobre $X$, por um isomorfismo $\left(i d_{X}, f\right):\left(X, \pi_{X}, X / G\right) \rightarrow(X, p, B)$, onde $i d_{X}: X \rightarrow X$ é a aplicação identidade e $f: X / G \rightarrow B$ é um homeomorfismo.

Definição 1.9.4 Um G-espaço $X$ é chamado um G-espaço principal se $X$ é um $G$-espaço livre com uma função transferência $t: X^{*} \rightarrow G$ contínua.

Um G-fibrado $\xi=(X, p, B)$, sobre um espaço base $B$, é chamado um G-fibrado principal se o seu espaço total $X$ é um G-espaço principal.

Observação 1.9.1 Se $\xi=(X, p, B)$ é um G-fibrado principal, sobre um espaço base $B$, então $\xi$ é um fibrado cuja fibra é o grupo $G$ e usamos a notação $\xi=(X, p, B, G)$. Em particular, se $X$ for um G-espaço principal, $\xi_{X}=$ $\left(X, \pi_{X}, X / G, G\right)$ é um G-fibrado principal.

Definição 1.9.5 Uma função contínua $h: X \rightarrow Y$ entre $G$-espaços $X$ e $Y$ é chamada uma aplicação G-equivariante, ou um G-morfismo, se $h(g x)=g h(x)$, para todo $x \in X$ e para todo $g \in G$. Uma aplicação G-equivariante $h: X \rightarrow Y$ induz, por passagem ao quociente, uma função contínua entre os espaços de órbitas

$$
\begin{array}{ll}
\bar{h}: X / G, & \rightarrow Y / G \\
G x=[x] & \mapsto G h(x)=[h(x)]
\end{array}
$$

chamada aplicação induzida por h, onde $G x$ e $G h(x)$ denotam as órbitas dos pontos $x$ e $h(x)$, respectivamente, pela ação de $G$.

\subsection{Espaços Classificantes}

Definição 1.10.1 Dado qualquer grupo topológico $G$, existe um espaço $B G$, chamado espaço classificante do grupo $G$, e um G-fibrado principal $p_{G}: E G \rightarrow$ 
$B G$, chamado o $G$-fibrado principal universal e denotado por $\omega_{G}=\left(E G, p_{G}, B G\right)$, onde EG é um espaço contrátil, tal que para qualquer espaço paracompacto Hausdorff $B$ existe uma bijeção entre o conjunto $[B, B G]$ das classes de homotopia de aplicações de $B$ em $B G$ e as classes de isomorfismos de G-fibrados principais sobre $B$.

Essa bijeção é definida associando-se a cada classe de homotopia em [B,BG], a qual é representada por uma função contínua $f: B \rightarrow B G$, o fibrado pullback $f^{*}\left(\omega_{G}\right)$.

Teorema 1.10.1 Para cada $G$-fibrado principal $\xi=(X, p, B, G)$ sobre um espaço paracompacto Hausdorff $B$, existe uma função contínua $c: B \rightarrow B G$ tal que $\xi$ e o fibrado pullback $c^{*}\left(\omega_{G}\right)$ são G-fibrados principais B-isomorfos. A função contínua $c: B \rightarrow B G$ é chamada uma aplicação classificante para o G-fibrado principal $\xi$.

Demonstração. Ver [19]

Teorema 1.10.2 Os fibrados vetoriais constituem uma classe especial de fibrados cujas fibras são espaços vetoriais. Mais precisamente, um fibrado vetorial real $n$-dimensional é um fibrado $\xi=\left(E, p, B, R^{n}\right)$ tal que para cada $b \in B, p^{-1}(b)$ possui uma estrutura de $R$-espaço vetorial n-dimensional e para uma trivialização local qualquer $\left(U_{\alpha}, \phi_{\alpha}\right)$ de $\xi$, as restrições

$$
\left.\phi_{\alpha}\right|_{p^{-1}}(b): p^{-1}(b) \rightarrow\{b\} \times R^{n}
$$

são isomorfismos de espaços vetoriais, para cada $b \in U_{\alpha}$.

Demonstração. Ver [19]

Na sequência, apresentamos um importante teorema para obtencão dos nossos resultados, cuja técnica da demostração envolve teoria de obstrução.

Teorema 1.10.3 Dado um fibrado vetorial temos que a classe de Euler é zero se, e somente se, existe uma seção não nula.

Demonstração. Ver [6]. 
Preliminares

\subsection{Fibrações}

Definição 1.11.1 Dizemos que uma função contínua $p: E \rightarrow B$, entre espaços topológicos E e B, tem a propriedade do levantamento de homotopia com relação a um espaço topológico $X$ se, dado uma homotopia $g_{t}: X \rightarrow B$ e uma função contínua $\tilde{g}_{0}: X \rightarrow E$ que é um levantamento de $g_{0}$, isto é, $p \circ \tilde{g}_{0}=g_{0}$, então existe uma homotopia $\tilde{g}_{t}: X \rightarrow E$ que levanta $g_{t}$, isto é, $p \circ \tilde{g}_{t}=g_{t}$.

Definição 1.11.2 Uma fibração é uma função contínua $p: E \rightarrow B$ que tem a propriedade do levantamento de homotopia com relação a qualquer espaço topológico $X$.

Observação 1.11.1 Seja $p: E \rightarrow B$ a projeção de um fibrado e suponha que o espaço base $B$ seja paracompacto. Então, $p: E \rightarrow B$ é uma fibração.

Observação 1.11.2 Todo fibrado cujo espaço base é uma variedade será uma fibração. Assim, quando trabalhamos com fibrados sobre variedades é comum o uso do termo fibração no lugar de fibrado.

\subsection{Sequência Espectral}

Definição 1.12.1 Um módulo diferencial bigraduado sobre um anel $R$, é uma coleção de R-módulos $\left\{E_{p, q}\right\}$ (ou $\left\{E^{p, q}\right\}$ ), para todo par de inteiros $p$ e q, junto com uma aplicação $R$-linear $d: E^{*, *} \rightarrow E^{*, *}$ o diferencial, de bigrau $(r,-r+1)$ (ou $d: E_{*, *} \rightarrow E_{*, *}$, de bigrau $(-r, r-1)$ ), para algum inteiro $r$, satisfazendo $d \circ d=0$.

As definições e propriedades apresentadas a seguir, também podem ser obtidas no caso de uma sequência espectral homológica mas consideraremos sequências espectrais do tipo cohomológicas até o final desta seção.

Definição 1.12.2 O módulo de cohomologia $H(E)$ é o módulo bigraduado

$$
H^{p, q}\left(E^{*, *}, d\right)=\frac{\operatorname{Ker}\left(d: E^{p, q} \rightarrow E^{p+r, q-r+1}\right)}{\operatorname{Im}\left(d: E^{p-r, q+r-1} \rightarrow E^{p, q}\right)}
$$


Definição 1.12.3 Uma sequência espectral do tipo cohomológico é uma coleção de $R$-módulos diferenciais bigraduados $\left\{E_{r}^{*, *}, d_{r}\right\}$ para $r=1,2, \cdots$ onde os diferenciais têm bigrau $(r,-r+1)$ e $E_{r+1}^{p, q}$ é isomorfo a $H^{p, q}\left(E_{r}^{*, *}, d_{r}\right)$.

Observação 1.12.1 Embora a sequência espectral esteja indexada para $r=$ $1,2, \cdots$, essa indexação pode começar em qualquer inteiro.

Para definir o termo limite de uma sequência espectral cohomológica, para todo $k \geq r$, denotemos por

$$
\begin{aligned}
& Z_{r}^{p, q}=\operatorname{Ker}\left(d_{r}: E_{r}^{p, q} \rightarrow E_{r}^{p+r, q-r+1}\right) \\
& B_{r}^{p, q}=\operatorname{Im}\left(d_{r}: E_{r}^{p-r, q+r-1} \rightarrow E_{r}^{p, q}\right)
\end{aligned}
$$

A condição $d_{r} \circ d_{r}=0$, implica que $B_{r} \subset Z_{r} \subset E_{r}$, e assim $E_{r+1} \cong Z_{r} / B_{r}$. Sejam

$$
\begin{gathered}
Z\left(E_{r+1}\right)^{p, q}=\operatorname{Ker}\left(d_{r+1}: E_{r+1}^{p, q} \rightarrow E_{r+1}^{p+r+1, q-r}\right) \\
B\left(E_{r+1}\right)^{p, q}=\operatorname{Im}\left(d_{r+1}: E_{r+1}^{p-r-1, q+r} \rightarrow E_{r+1}^{p, q}\right)
\end{gathered}
$$

existem submódulos bigraduados $Z_{r+1}$ e $B_{r+1}$ de $Z_{r}$, contendo $B_{r}$, tais que $Z\left(E_{r+1}\right)^{p, q} \cong Z_{r+1}^{p, q} / Z_{r}^{p, q}$ e $B\left(E_{r+1}\right)^{p, q} \cong B_{r+1}^{p, q} / B_{r}^{p, q}$ para todo $p, q$. Assim, $B_{r+1} \subset$ $Z_{r+1}$ e temos que

$$
B_{r} \subset B_{r+1} \subset Z_{r+1} \subset Z_{r} \subset E_{r}
$$

Além disso, $E_{r+2} \cong Z\left(E_{r+1}\right) / B\left(E_{r+1}\right) \cong Z_{r+1} / B_{r+1}$. Continuando esse processo por indução, obtemos uma sequência de submódulos, para todo $n \geq r$,

$$
B_{r} \subset B_{r+1} \subset \cdots \subset B_{n} \subset \cdots \subset Z_{n} \subset \cdots \subset Z_{r+1} \subset Z_{r} \subset E_{r}
$$

com a propriedade que $E_{n+1} \cong Z_{n} / B_{n}$. 
Definição 1.12.4 Definimos os módulos bigraduados

$$
Z_{\infty}=\bigcap_{n} Z_{n} \quad \text { e } \quad B_{\infty}=\bigcup_{n} B_{n}
$$

O módulo bigraduado $E_{\infty}=Z_{\infty} / B_{\infty}$ é chamado o limite da sequência espectral E.

Definição 1.12.5 Uma sequência espectral cohomológica $\left\{E_{r}^{*, *}, d_{r}\right\}$ colapsa no $N$-ésimo termo se o diferencial $d_{r}=0$, para todo $r \geq N$.

Observação 1.12.2 Uma consequência imediata do fato de uma sequência espectral cohomológica $\left\{E_{r}^{* * *}, d_{r}\right\}$ colapsar no $N$-ésimo termo,é que $E_{N}^{*, *} \cong E_{N+1}^{*, *} \cong$ $\cdots \cong E_{\infty}^{*, *}$.

Definição 1.12.6 Uma filtração decrescente $F$ sobre um R-módulo A, é uma família de submódulos $\left\{F^{p}(A)\right\}$, com $p \in \mathbb{Z}$, tal que

$$
\cdots \subset F^{p+1}(A) \subset F^{p}(A) \subset F^{p-1}(A) \subset \cdots \subset A
$$

Definição 1.12.7 Dada uma filtração decrescente $F$ sobre um $R$-módulo $A$, o módulo graduado associado $E_{0}^{*}(A)$ é dado por

$$
E_{0}^{p}(A)=F^{p}(A) / F^{p+1}(A)
$$

Definição 1.12.8 Se $H^{*}$ é um $R$-módulo graduado e se F é uma filtração sobre $H^{*}$, então $F^{p}\left(H^{n}\right)=F^{p}\left(H^{*}\right) \cap H^{n} \subset F^{p-1}\left(H^{*}\right) \cap H^{n}=F^{p-1}\left(H^{n}\right)$ e o módulo bigraduado associado $E_{0}^{*, *}$ é dado por

$$
E_{0}^{p, q}\left(H^{*}, F\right)=F^{p}\left(H^{p+q}\right)=F^{p+1}\left(H^{p+q}\right)
$$

Definição 1.12.9 Uma sequência espectral $\left\{E_{r}^{*, *}, d_{r}\right\}$ converge para um $R$ módulo graduado $H^{*}$, se existe uma filtração $F$ sobre $H^{*}$ tal que

$$
E_{\infty}^{p, q} \cong E_{0}^{p, q}\left(H^{*}, F\right)
$$


onde $E_{\infty}^{*, *}$ é o termo limite da sequência espectral.

Definição 1.12.10 Uma sequência espectral $\left\{E_{r}^{*, *}, d_{r}\right\}$ é uma sequência espectral do primeiro quadrante, se existe $r$ tal que $E_{r}^{p, q}=0$, para $p<0$ ou $q<0$.

Definição 1.12.11 (A Sequência Espectral Cohomológica de Leray-Serre). Seja $R$ um anel comutativo com unidade. Dada uma fibração $F \hookrightarrow E \stackrel{p}{\longrightarrow} B$ onde $B$ é conexo por caminhos, existe uma sequência espectral do primeiro quadrante $\left\{E_{r}^{*, *}, d_{r}\right\}, c o m$

$$
E_{2}^{p, q} \cong H^{p}\left(B ; \mathrm{H}^{q}(F ; R)\right),
$$

a cohomologia de B com coeficientes locais na cohomologia de F, a fibra de $p$, e convergindo para $H^{*}(E ; R)$. Além disso, essa sequência é natural com relação a aplicações entre fibrações que preservem fibras.

\subsection{Produto semi-direto}

Considere $H$ e $Q$ dois grupos e $\theta: Q \rightarrow A u t(H)$ um homomorfismo de grupos.

Definição 1.13.1 O produto semi-direto $H \rtimes_{\theta} Q$ é definido como o conjunto $\{(h, q) \mid h \in H, q \in Q\}$ com a operação de grupos $(h, q)\left(h^{\prime}, q^{\prime}\right)=\left(h \theta(q)\left(h^{\prime}\right), q q^{\prime}\right)$.

Observação 1.13.1 O elemento inverso de $(h, q)$ é $\left(\theta\left(q^{-1}\right)\left(h^{-1}\right), q^{-1}\right)$.

Considere $G=H \rtimes Q$, existem monomorfismos canônicos $H \rightarrow G$ e $Q \rightarrow G$ definidos por

$$
\begin{cases}h \rightarrow\left(h, 1_{Q}\right) & h \in H \\ q \rightarrow\left(1_{H}, q\right) & q \in Q\end{cases}
$$

onde $1_{H}\left(\right.$ resp. $\left.1_{Q}\right)$ é o elemento identidade de $H(\operatorname{resp} . Q)$.

Estes monomorfismos são naturais logo podemos tratar $H$ e $Q$ como subgrupos de $G$ por estas inclusões. 
Teorema 1.13.1 Seja $G=H \rtimes Q$, então:

a) $H$ é subgrupo normal de $G$

b) $H Q=G$

c) $H \cap Q=\left\{1_{G}\right\}$

Demonstração. Seja $p: G \rightarrow Q$ a projeção de finida por $p(h, q)=q$. Então $p$ é um homomorfismo de kernel $H$, logo $H$ é um subgrupo normal de $G$.

Todo $(h, q) \in G$ pode ser escrito como $\left(h, 1_{Q}\right)\left(1_{H}, q\right)$, portanto $H Q=G$.

Se $(h, q) \in H \cap Q \Longrightarrow\left\{\begin{array}{l}(h, q) \in H ; \\ (h, q) \in Q .\end{array} \Longrightarrow\left\{\begin{array}{l}(h, q)=\left(h, 1_{Q}\right) ; \\ (h, q)=\left(1_{H}, q\right) .\end{array} \Longrightarrow(h, q)=\right.\right.$ $\left(1_{H}, 1_{Q}\right)=1_{G}$ 


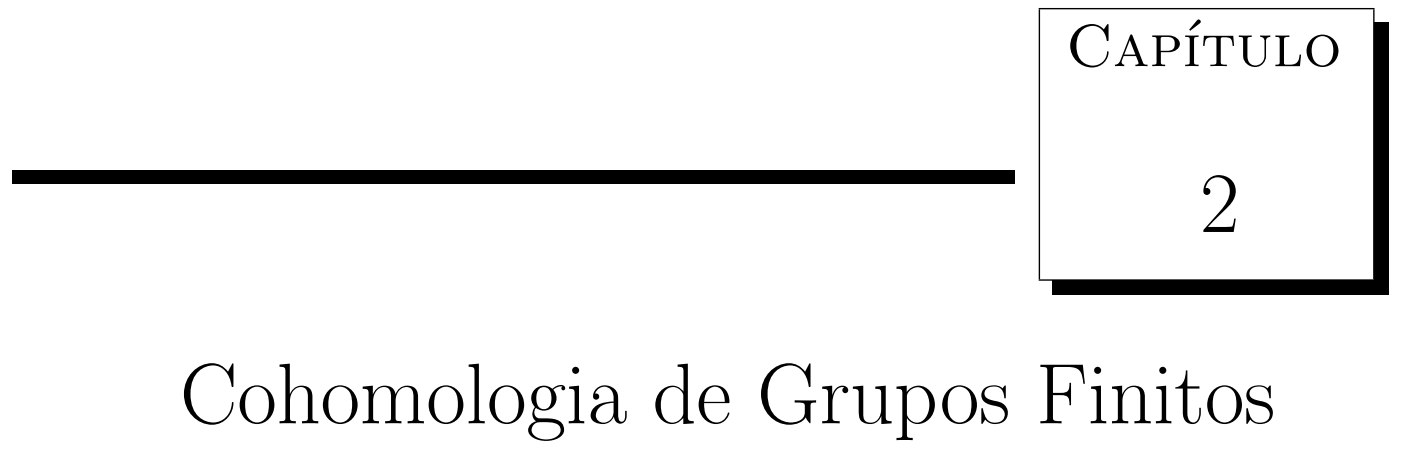

Neste capítulo apresentamos a cohomologia de um grupo finito e como tal cohomologia se relaciona diretamente com a cohomologia dos espaços de forma esférica. Mais especificamente, na seção 2.3 apresentamos o anel de cohomologia módulo $p$ dos grupos períodicos finitos.

\subsection{Grupos atuando livremente em esferas}

Nesta seção apresentamos algumas propriedades interessantes da ação livre de um grupo finito em uma esfera.

Proposição 2.1.1 Sejam $X$ um $C W$-complexo compacto e $G$ um grupo propriamente descontínuo de homeomorfismos de $X$. Se a ação de $G$ em $X$ é livre, então $G$ é finito.

Demonstração. Seja $X / G$ o espaço de órbitas de $X$. Temos que

$$
\begin{aligned}
p: X & \rightarrow X / G \\
x & \mapsto \bar{x}
\end{aligned}
$$


é recobrimento.

Agora, dado $\bar{x}_{0} \in X / G$, temos

$$
\begin{aligned}
p^{-1}\left(\bar{x}_{0}\right) & =\left\{x \in X ; p(x)=\bar{x}_{0}\right\} \\
& =\left\{x \in X ; \bar{x}=\bar{x}_{0}\right\} \\
& =\left\{x \in X ; x \in G\left(x_{0}\right)\right\} \\
& =G\left(x_{0}\right) .
\end{aligned}
$$

Como a ação de $G$ em $X$ é livre, $p^{-1}\left(\bar{x}_{0}\right)$ está em correspondência 1-1 com $G$. Além disso, a fibra $p^{-1}\left(\bar{x}_{0}\right)$ é fechada e discreta e sendo $X$ compacto segue que $p^{-1}\left(\bar{x}_{0}\right)$ é um conjunto finito. Portanto $G$ é finito.

Proposição 2.1.2 O grupo $\mathbb{Z}_{2}$ é o único grupo não trivial que atua livremente em uma esfera de dimensão par $S^{2 k}$.

Demonstração. Sejam $G$ um grupo atuando livremente em $S^{2 k}$ e $f, g \neq i d$.

Como a ação é livre, temos que $f$ não tem pontos fixos e ainda, como $f$ é um homeomorfismo, temos $f \circ f^{-1}=i d$. Portanto $\operatorname{deg}(f) \cdot \operatorname{deg}\left(f^{-1}\right)=1$ e assim $\operatorname{deg}(f)= \pm 1$. Mais ainda, $\operatorname{deg}(f)=-1$, visto que se $\operatorname{deg}(f)=1$, então o número de Lefschetz

$$
\begin{aligned}
\Lambda(f) & =\sum_{k=0}^{\infty}(-1)^{k} \operatorname{tr}\left(f_{k}\right) \\
& =\sum_{k=0}^{2 k}(-1)^{k} \operatorname{tr}\left(f_{k}\right) \\
& =(-1)^{0} \operatorname{tr}\left(f_{0}\right)+(-1)^{2 k} \operatorname{tr}\left(f_{2 k}\right) \\
& =1 \operatorname{deg}(f)+(-1)^{2 k} \operatorname{deg}(f) \\
& =1+(-1)^{2 k} \neq 0 .
\end{aligned}
$$

pois $S^{2 k-1}=e^{0} \cup e^{2 k-1}$, o que implica que $f$ tem pontos fixos .

Agora, $\operatorname{deg}(f \circ f)=\operatorname{deg}(f) \cdot \operatorname{deg}(f)=\operatorname{deg}\left(f^{2}\right)=1$ ou seja, $\Lambda\left(f^{2}\right) \neq 0$ e portanto $f^{2}$ tem ponto fixo.

Como a ação é livre segue que $f^{2}=i d$. Analogamente $g^{2}=i d$. 
Assim, se $\operatorname{deg}(f \circ g)=1$ então $\Lambda(f \circ g) \neq 0$ e assim $f \circ g=i d$, contudo $f^{2}=i d=g^{2}$, concluímos então que $f=g^{-1}=f^{-1}=g$. Logo $f=g$ e assim $G \approx \mathbb{Z}_{2}$.

Observação 2.1.1 Segue da proposição anterior que se $G$ é um grupo não trivial, $G \neq \mathbb{Z}_{2}$, atuando livremente em uma esfera $S^{m}$ então $m$ é ímpar.

Proposição 2.1.3 Seja $X$ um G-complexo livre homeomorfo a uma esfera de dimensão impar $S^{2 k-1}$. Então a ação de $G$ sobre $H_{2 k-1}(X) \simeq \mathbb{Z}$ é trivial.

Demonstração. Ver [19].

Proposição 2.1.4 Seja G um grupo finito agindo livremente sobre a esfera $S^{n}$ de dimensão n. A fim de obter uma resolução para o grupo $G$ é suficiente obter uma decomposição celular G-equivariante para $S^{n}$.

Demonstração. Ver [18].

Como consequência obtemos o seguinte corolário.

Corolário 2.1.1 Seja G um grupo finito agindo livremente sobre a esfera $S^{n}$ de dimensão n. Então $H^{l}\left(S^{n} / G ; \mathbb{Z}_{p}\right)=H^{l}\left(G ; \mathbb{Z}_{p}\right)$ para $l \leq n$.

\subsection{Grupos com Cohomologia Periódica}

Nesta seção apresentamos um estudo sobre a cohomologia de grupos periódicos finitos que será de fundamental importância para os próximos capítulos. Particularmente, a teoria de ação de grupos finitos em esferas está intimamente relacionada com a

Teorema 2.2.1 Seja $X$ um G-complexo livre homeomorfo a uma esfera de dimensão impar $S^{2 k-1}$. Considere o complexo de cadeia celular aumentado de $X, C_{*}(X) \stackrel{\varepsilon}{\longrightarrow} \mathbb{Z}$. Então, a sequência

$$
\cdots \rightarrow C_{2 k-1}(X) \rightarrow \cdots \rightarrow C_{1}(X) \stackrel{\partial_{f}}{\rightarrow} C_{0}(X) \stackrel{n \circ \varepsilon}{\rightarrow} C_{2 k-1}(X) \stackrel{\partial_{2 k-1}}{\rightarrow} \cdots \rightarrow C_{0}(X) \stackrel{\varepsilon}{\rightarrow} \mathbb{Z} \rightarrow 0,
$$


onde $n: \mathbb{Z}=H_{2 k-1}(X) \rightarrow C_{2 k-1}(X)$ é a inclusão, é uma resolução livre de $\mathbb{Z}$ sobre $\mathbb{Z} G$ que é periódica de período $2 k$.

Demonstração. Ver [19].

Exemplo 2.2.1 Se $G=<t>\simeq \mathbb{Z}_{n}$ é um grupo cíclico finito de ordem $n$, então:

$$
H_{i}(G ; \mathbb{Z})=\left\{\begin{array}{l}
\mathbb{Z}, \text { se } i=0 ; \\
\mathbb{Z}_{n}, \text { se } i \text { é ímpar; } \\
0, \text { se } i \text { é par. }
\end{array} \quad \begin{array}{l}
\mathbb{Z}, \text { se } i=0 \\
\mathbb{Z}_{n}, \text { se } i \text { é par } ; \\
0, \text { se } i \text { é ímpar } .
\end{array}\right.
$$

Definição 2.2.1 Um grupo finito $G$ tem cohomologia periódica, ou seja, $G$ é dito periódico de período $k$ se $H^{i}(G, M) \cong H^{i+k}(G, M)$ para todo $i \geq 1$.

Proposição 2.2.1 [1, Lema 6.2, cap. IV] Se $G$ é um grupo finito que atua livremente em um $C W$-complexo $X$ homeomorfo a uma esfera de dimensão ímpar $S^{2 k-1}$, então $G$ tem cohomologia periódica de período $2 k$.

\subsection{Anel de Cohomologia de Grupos Periódicos}

Nesta seção usamos [1] como principal referência.

Teorema 2.3.1 Seja $H \subset G$ um subgrupo normal e considere a sobrejeção induzida

$$
B_{p}: B_{G} \rightarrow B_{G / H}
$$

Existe uma sequência espectral convergindo para $H^{*}(G ; A)$ para coeficientes sem torção A com $E_{2}^{i, j}$-termo $H^{i}\left(G / H ; H_{j}^{i}(H ; A)\right)$. Esta sequência é a sequência espectral de Serre chamada também de sequência espectral de Lyndon-HochschildSerre.

Demonstração. Ver [1] 
Definição 2.3.1 Dados $N \triangleleft G$ e $\pi: G \rightarrow G / N$. Dizemos que $N \stackrel{\triangleleft}{\rightarrow} G \stackrel{\pi}{\rightarrow} G / N$ é uma extensão central.

Lema 2.3.1 Considere uma extensão central $\mathbb{Z}_{p} \stackrel{\triangleleft}{\rightarrow} E \stackrel{\pi}{\rightarrow} G$ e a fibração dos espaços classificantes

$$
B_{\mathbb{Z}_{p}} \stackrel{j}{\rightarrow} B_{E} \stackrel{B_{\pi}}{\rightarrow} B_{G}
$$

Então existe um $n$ finito tal que $E_{n}=E \infty$ na sequência espectral da fibração .

Demonstração. A aplicação de Frobenius associada a inclusão $\mathbb{Z} \hookrightarrow E$ dá origem a um homomorfismo $\varphi: E \rightarrow \mathbb{Z}_{p} \prec S_{|G|}$ (produto wreath, para mais detalhes ver [1] )e assim $\varphi^{*}(b \otimes \cdots \otimes b)(|G|-$ vezes $)$ é não nula e se restringe a $b^{|G|} \mathrm{em}$ $H^{*}\left(\mathbb{Z}_{p} ; \mathbb{Z}_{p}\right)$ pois $\mathbb{Z}_{p}$ é central em E. Logo a classe $b^{|G|}$ em $E_{2}^{0,2|G|}$ é um ciclo infinito na sequência espectral. Assim $E_{2}=\mathbb{Z}_{p}\left[b^{|G|}\right] \otimes\left(E_{2}^{*, 0} \otimes \cdots \otimes E_{2}^{*, 2|G|-1}\right)$ e por indução temos que $E_{2|G|}=E_{\infty}$

A sequência espectral de Lyndon-Hochschild-Serre para $\star$ tem $E_{2}$-termo $H^{*}\left(G ; \mathbb{Z}_{p}\right) \otimes H^{*}\left(\mathbb{Z}_{p} ; \mathbb{Z}_{p}\right)$ e, existe uma aplicação da sequência espectral de Serre da fibração

$$
B_{\mathbb{Z}_{p}} \stackrel{j}{\rightarrow} E\left(B_{E}\right) \stackrel{B_{\pi}}{\rightarrow}\left(B_{G}\right)
$$

com $E_{2}$-termo $H^{*}\left(K\left(\mathbb{Z}_{p}, 2\right) ; \mathbb{Z}_{p}\right) \otimes H^{*}\left(\mathbb{Z}_{p} ; \mathbb{Z}_{p}\right)$ para a sequência espectral da extensão devido a naturalidade da sequência espectral de Serre e da comutatividade do diagrama de fibrações

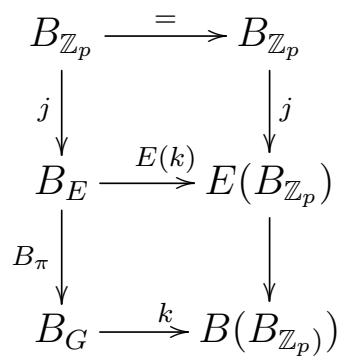

Em particular a aplicação é a identidade em $H^{*}\left(\mathbb{Z}_{p} ; \mathbb{Z}_{p}\right)$ e é a aplicação

$$
k^{*}: H^{*}\left(K\left(\mathbb{Z}_{p}, 2\right) ; \mathbb{Z}_{p}\right) \rightarrow H^{*}\left(G ; \mathbb{Z}_{p}\right)
$$


Essa aplicação também comuta com os diferenciais, assim como os produtos cup devido à naturalidade e consequentemente os diferenciais na sequência espectral de $K\left(\mathbb{Z}_{p}, 2\right)$ implicam em diferenciais na sequência espectral de LyndonHochschild-Serre da extensão central.

Na sequência espectral de $K\left(\mathbb{Z}_{p}, 2\right)$ os diferenciais são dados por $d_{2}\left(e_{1}\right)=\iota_{2}$ então $d_{3}(b)=\beta\left(\iota_{2}\right)$. Depois disso os diferenciais são dados por $d_{2 p^{i}+1}\left(b^{p^{i}}\right)=$ $P^{p^{i-1}} \cdot P^{p^{i-2}} \cdots P^{1} \beta\left(\iota_{2}\right)$, onde $\beta$ é o Bockstein e $P$ é um polinômio. Quando $p=2$ estes são todos os diferenciais.

Se $p$ é ímpar então existem também os seguintes diferenciais $d_{2 p-1}\left(b^{p-1} \beta\left(\iota_{2}\right)\right)=$ $\beta P^{1} \beta\left(\iota_{2}\right)$ e mais geralmente $d_{2 p^{i}(p-1)+1}\left(b^{p^{i}(p-1)} d_{2 p^{i}+1} b^{p^{i}}\right)=\beta d_{p^{i+1}+1}\left(b^{p^{i+1}}\right)$.

Teorema 2.3.2 [1, Corolário 6.8 cap IV] Se G é um grupo periódico então $H^{*}\left(G ; \mathbb{Z}_{p}\right)=\mathbb{Z}_{p}\left[b^{i}\right] \otimes E\left(e_{2 i-1}\right)$ para $i$ impar, onde $i$ divide $(p-1)$.

Observação 2.3.1 No teorema anterior $\mathbb{Z}_{p}\left[b^{i}\right]$ é uma álgebra polinomial com gerador $2 i$-dimensional e $E\left(e_{2 i-1}\right)$ é uma álgebra exterior com gerador 1dimensional. 


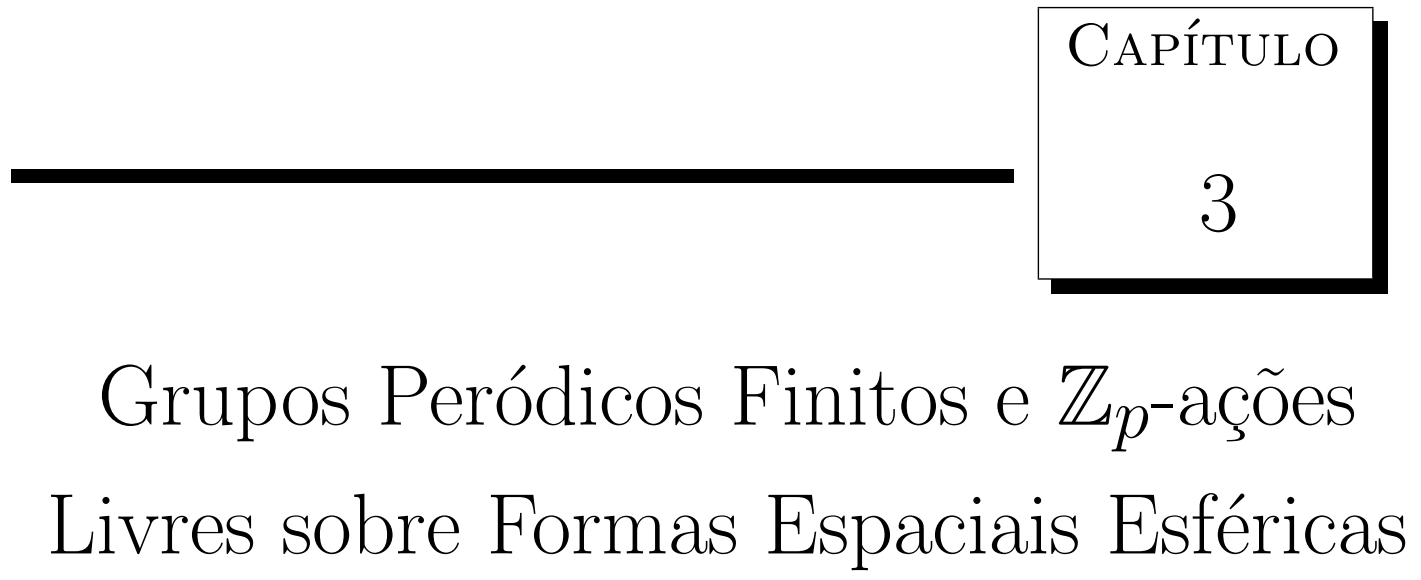

Neste capítulo nós apresentamos uma caracterização das formas espaciais esféricas que admitem $\mathbb{Z}_{p}$-ações livres (ver Teorema 3.1.1). A existência de tais $\mathbb{Z}_{p}$-espaços livres está diretamente relacionada com os subgrupos de índice primo dos grupos periódicos finitos. Na seção 3.2. nosso objetivo foi assegurar a existência de tais subgrupos, e desta forma, garantir a existência de espaços de forma esférica que admitem uma ação livre de $\mathbb{Z}_{p}$.

\section{1 $\mathbb{Z}_{p}$-ações Livres sobre Formas Espaciais Esféricas}

Proposição 3.1.1 Seja $\Sigma^{m}$ um $C W$-complexo m-dimensional com o mesmo tipo de homotopia da m-esfera. Dada uma ação celular livre $\gamma$ de um grupo finito $G$ em $\Sigma^{m}$, seja $\Sigma^{m} / \gamma(G)$ a forma espacial esferica homotópica m-dimensional, então $\pi_{1}\left(\Sigma^{m} / \gamma(G)\right) \simeq G$. 
Demonstração. Temos que $\pi: \Sigma^{m} \rightarrow \Sigma^{m} / \gamma(G)$ é um recobrimento universal. Da Proposição 1.2 .5 temos $G=A\left(\Sigma^{m}, \pi\right)$ e assim pela Proposição 1.2 .6 temos $\pi_{1}\left(\Sigma^{m} / \gamma(G)\right) \simeq A\left(\Sigma^{m}, \pi\right)$.

Portanto $\pi_{1}\left(\Sigma^{m} / \gamma(G)\right) \simeq G$.

Considere a tabela 3.1 de todos os grupos periódicos finitos, onde:

\begin{tabular}{|c|c|c|}
\hline Tipo & Definição & Condição \\
\hline I & $\mathbb{Z}_{a} \rtimes_{\phi} \mathbb{Z}_{b}$ & $(a, b)=1$ \\
\hline II & $\mathbb{Z}_{a} \rtimes_{\phi}\left(\mathbb{Z}_{b} \times Q_{2^{t}}\right)$ & $(a, b)=(a b, 2)=1$ \\
\hline III & $\mathbb{Z}_{a} \rtimes_{\phi}\left(\mathbb{Z}_{b} \times T_{i}\right)$ & $(a, b)=(a b, 6)=1$ \\
\hline IV & $\mathbb{Z}_{a} \rtimes_{\phi}\left(\mathbb{Z}_{b} \times O_{i}^{*}\right)$ & $(a, b)=(a b, 6)=1$ \\
\hline V & $\left(\mathbb{Z}_{a} \rtimes_{\phi} \mathbb{Z}_{b}\right) \times S L_{2}\left(\mathbb{F}_{r}\right)$ & $(a, b)=\left(a b, r\left(r^{2}-1\right)\right)=1$ \\
\hline VI & $\mathbb{Z}_{a} \rtimes_{\phi}\left(\mathbb{Z}_{b} \times T L_{2}\left(\mathbb{F}_{q}\right)\right)$ & $(a, b)=\left(a b, q\left(q^{2}-1\right)\right)=1$ \\
\hline
\end{tabular}

Tabela 3.1: Grupos Periódicos Finitos

- $\phi: G \rightarrow A u t(H)$ e $H \rtimes_{\phi} G$ é o conjunto $H \times G$ com o produto definido por $(h, g)\left(h^{\prime}, g^{\prime}\right)=\left(h \phi(g)\left(h^{\prime}\right), g g^{\prime}\right)$.

- $t \geq 3, \quad i \geq 1$ e $r$ e $q$ são números primos tais que $r \geq 3, \quad q \geq 5$.

- os grupos $Q_{2^{t}}=\left\langle x, y: x^{2^{t-1}}=y^{2}, \quad y x y^{-1}=x^{-1}\right\rangle$ são os quaterniônicos generalizados e os grupos $T_{i}$ e $O_{i}^{*}$ são o tetraedral binário e o octaedral binário generalizados.

Teorema 3.1.1 Se uma forma espacial esférica homotópica $\Sigma^{m} / \eta(H)$ admite uma ação livre de $\mathbb{Z}_{p}$, então $H$ é um subgrupo de indice $p$ de algum grupo $G$ da tabela 3.1. Reciprocamente, se $H$ é um subgrupo normal de índice $p$ de algum grupo $G$ da tabela 3.1, então existe uma forma espacial esférica homotópica com grupo fundamental $H$ e ação livre de $\mathbb{Z}_{p}$.

Demonstração. Suponha uma ação livre de $\mathbb{Z}_{p}$ em $\Sigma^{m} / \eta(H)=X$. Então temos o recobrimento $q: X \rightarrow X / \mathbb{Z}_{p}$ à $p$-folhas. Assim $\left[\pi_{1}\left(X / \mathbb{Z}_{p}\right): q_{*}\left(\pi_{1}(X)\right)\right]=p$. Por 
outro lado, segue da Proposição 3.1.1 que

$$
\pi_{1}(X)=\pi_{1}\left(\Sigma^{m} / \eta(H)\right) \simeq H
$$

Como $q_{*}: \pi_{1}(X) \rightarrow \pi_{1}\left(X / \mathbb{Z}_{p}\right)$ é injetor, então $i m\left(q_{*}\right)=q_{*}\left(\pi_{1}(X)\right) \simeq \pi_{1}(X) \simeq$ H. Logo, $\left[\pi_{1}\left(X / \mathbb{Z}_{p}\right): H\right]=p$.

Agora, considerando $G=\pi_{1}\left(X / \mathbb{Z}_{p}\right)$, basta mostrar então, que $G$ é um dos grupos da tabela 3.1 .

Considere $\Pi: \Sigma^{m} \rightarrow \Sigma^{m} / \eta(H)=X$, e a seguinte composição

$$
\Sigma^{m} \stackrel{\Pi}{\longrightarrow} \Sigma^{m} / \eta(H)=X \stackrel{q}{\longrightarrow} X / \mathbb{Z}_{p}
$$

Assim temos o seguinte recobrimento $q \circ \Pi: \Sigma^{m} \rightarrow X / \mathbb{Z}_{p}$ com fibra $G$ (ver [15, Corolário 2, pag. 169] e [15, Proposição 11, pag. 171] ). Isto significa que $\left(\Sigma^{m} / \eta(H)\right) / \mathbb{Z}_{p}=\Sigma^{m} / \gamma(G)$ para algum $\gamma$. Portanto, $G$ deve ser um dos grupos da tabela 3.1 .

Reciprocamente, suponha $H \triangleleft G$ subgrupo normal de índice $p$, para algum $H$ e $G$ na tabela 3.1.

Como G é um grupo da tabela 3.1, existe uma ação livre $\gamma$ de $G$ sobre $\Sigma^{m}$ e portanto $q: \Sigma^{m} \rightarrow \Sigma^{m} / \gamma(G)$ é um $G$-fibrado principal.

Assim a restrição $\gamma_{H}$ de $\gamma$ em $H$ define uma ação livre de $H$ sobre $\Sigma^{m}$ e $\pi_{1}\left(\Sigma^{m} / \gamma_{H}(H)\right) \simeq H$. Logo, de [17, Lema 1.2.11] existe uma $\mathbb{Z}_{p} \simeq G / H$ - ação livre sobre $\Sigma^{m} / \gamma_{H}(H)$.

Proposição 3.1.2 Sejam G e H dois grupos da tabela 3.1 de período pn e assuma que $H$ é um subgrupo de $G$ de indice $p$. Então o homomorfismo induzido $B_{i}^{p n}$ : $H^{p n}(B G, \mathbb{Z}) \rightarrow H^{p n}(B H, \mathbb{Z})$ é sobrejetor.

Demonstração. A inclusão $i: H \hookrightarrow G$ induz um homomorfismo $(i)^{p n}$ : $H^{p n}(G, \mathbb{Z}) \rightarrow H^{p n}(H, \mathbb{Z})$. Temos o homomorfismo transfer $\operatorname{tr}_{H}^{G}: H^{p n}(H, \mathbb{Z}) \rightarrow$ $H^{p n}(G, \mathbb{Z})$ e a composição $t r_{H}^{G} \circ i^{p n}$ é uma multiplicação por $p$. 


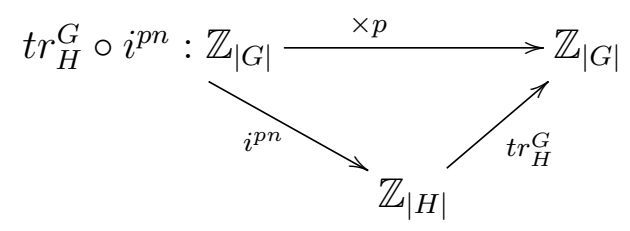

Como $H^{p n}(G, \mathbb{Z})$ e $H^{p n}(H, \mathbb{Z})$ são grupos cíclicos de ordem $|G|$ e $|H|$ respectivamente e $|G|=p|H|$, temos $\operatorname{Im}\left(\operatorname{tr}_{H}^{G} \circ i^{p n}\right)=\mathbb{Z}_{|G|}$. Logo $(i)^{p n}$ é sobrejetora.

\subsection{Subgrupos de Índice Primo}

Nesta seção vamos calcular alguns subgrupos de índice $p$ primo de grupos periódicos finitos.

\section{Tipo I}

Considere o grupo $\mathbb{Z}_{a} \rtimes_{\phi} \mathbb{Z}_{b}$ do Tipo I da Tabela 3.1. No nosso caso devemos encontrar subgrupos normais de índice primo $p$ deste grupo.

Seja $b$ um número da forma $b=p \cdot p_{1}^{\alpha_{1}} \cdot p_{2}^{\alpha_{2}} \cdot p_{3}^{\alpha_{3}} \cdots p_{n}^{\alpha_{n}} \operatorname{com} p_{i}$ primo, $(a, b)=1$, $p_{i} \neq p_{j}$ e $p \neq p_{j} \forall i, j$.

Logo $\mathbb{Z}_{b}$ pode ser escrito da forma $\mathbb{Z}_{b}=\mathbb{Z}_{p} \times \mathbb{Z}_{p_{1}^{\alpha_{1}}} \times \mathbb{Z}_{p_{2}^{\alpha_{2}}} \times \mathbb{Z}_{p_{3}^{\alpha_{3}}} \times \cdots \times$ $\mathbb{Z}_{p_{n}^{\alpha_{n}}}$ (Teorema Chinês do Resto para anéis).

$\operatorname{Assim} \mathbb{Z}_{a} \rtimes_{\phi} \mathbb{Z}_{b}=\mathbb{Z}_{a} \rtimes_{\phi}\left(\mathbb{Z}_{p} \times \mathbb{Z}_{p_{1}^{\alpha_{1}}} \times \mathbb{Z}_{p_{2}^{\alpha_{2}}} \times \mathbb{Z}_{p_{3}^{\alpha_{3}}} \times \cdots \times \mathbb{Z}_{p_{n}^{\alpha_{n}}}\right)$, com ordem $a b=a \cdot p \cdot p_{1}^{\alpha_{1}} \cdot p_{2}^{\alpha_{2}} \cdot p_{3}^{\alpha_{3}} \cdots p_{n}^{\alpha_{n}}$.

Considere a seguinte aplicação:

$$
\begin{aligned}
f: \mathbb{Z}_{a} \rtimes_{\phi}\left(\mathbb{Z}_{p} \times \mathbb{Z}_{p_{1}^{\alpha_{1}}} \times \mathbb{Z}_{p_{2}^{\alpha_{2}}} \times \mathbb{Z}_{p_{3}^{\alpha_{3}}} \times \cdots \times \mathbb{Z}_{p_{n}^{\alpha_{n}}}\right) & \rightarrow \mathbb{Z}_{p} \\
\left(a_{1},\left(p^{\prime}, p_{1}, p_{2}, \cdots, p_{n}\right)\right) & \longrightarrow p^{\prime}
\end{aligned}
$$

onde $a_{1} \in \mathbb{Z}_{a}, p^{\prime} \in \mathbb{Z}_{p}$ e $p_{i} \in \mathbb{Z}_{p_{i}^{\alpha_{i}}}$.

Temos que $f$ é um homomorfismo de kernel $\mathbb{Z}_{a} \rtimes_{\phi}\left(\left\{1_{\mathbb{Z}_{p}}\right\} \times \mathbb{Z}_{p_{1}^{\alpha_{1}}} \times \mathbb{Z}_{p_{2}^{\alpha_{2}}} \times \mathbb{Z}_{p_{3}^{\alpha_{3}}} \times\right.$ $\left.\cdots \times \mathbb{Z}_{p_{n}^{\alpha_{n}}}\right)$ que é isomorfo a $\mathbb{Z}_{a} \rtimes_{\psi}\left(\mathbb{Z}_{p_{1}^{\alpha_{1}}} \times \mathbb{Z}_{p_{2}^{\alpha_{2}}} \times \mathbb{Z}_{p_{3}^{\alpha_{3}}} \times \cdots \times \mathbb{Z}_{p_{n}^{\alpha_{n}}}\right)$, onde $\psi=\phi \circ i$ com $i: \mathbb{Z}_{p_{1}^{\alpha_{1}}} \times \mathbb{Z}_{p_{2}^{\alpha_{2}}} \times \mathbb{Z}_{p_{3}^{\alpha_{3}}} \times \cdots \times \mathbb{Z}_{p_{n}^{\alpha_{n}}} \longrightarrow \mathbb{Z}_{p} \times \mathbb{Z}_{p_{1}^{\alpha_{1}}} \times \mathbb{Z}_{p_{2}^{\alpha_{2}}} \times \mathbb{Z}_{p_{3}^{\alpha_{3}}} \times \cdots \times \mathbb{Z}_{p_{n}^{\alpha_{n}}}$ definida por $\left(p_{1}, p_{2}, \cdots, p_{n}\right) \longrightarrow\left(1_{\mathbb{Z}_{p}}, p_{1}, p_{2}, \cdots, p_{n}\right)$. 


\begin{tabular}{|c|c|c|}
\hline Tipo & Subgrupo & Condição \\
\hline I & $\mathbb{Z}_{a} \rtimes_{\psi} \mathbb{Z}_{b_{p}}$ & $(a, b)=1 ; \quad\left(p, b_{p}\right)=1$ \\
\hline II & $\mathbb{Z}_{a} \rtimes_{\psi}\left(\mathbb{Z}_{b_{p}} \times Q_{2^{t}}\right)$ & $(a, b)=(a b, 2)=1 ; \quad\left(p, b_{p}\right)=1$ \\
\hline III & $\mathbb{Z}_{a} \rtimes_{\psi}\left(\mathbb{Z}_{b_{p}} \times T_{i}\right)$ & $(a, b)=(a b, 6)=1 ; \quad\left(p, b_{p}\right)=1$ \\
\hline IV & $\mathbb{Z}_{a} \rtimes_{\psi}\left(\mathbb{Z}_{b_{p}} \times O_{i}^{*}\right)$ & $(a, b)=(a b, 6)=1 ; \quad\left(p, b_{p}\right)=1$ \\
\hline V & $\left(\mathbb{Z}_{a} \rtimes_{\psi} \mathbb{Z}_{b_{p}}\right) \times S L_{2}\left(\mathbb{F}_{r}\right)$ & $(a, b)=\left(a b, r\left(r^{2}-1\right)\right)=1 ; \quad\left(p, b_{p}\right)=1$ \\
\hline VI & $\mathbb{Z}_{a} \rtimes_{\psi}\left(\mathbb{Z}_{b_{p}} \times T L_{2}\left(\mathbb{F}_{q}\right)\right)$ & $(a, b)=\left(a b, q\left(q^{2}-1\right)\right)=1 ; \quad\left(p, b_{p}\right)=1$ \\
\hline
\end{tabular}

Tabela 3.2: Grupos de Índice Primo

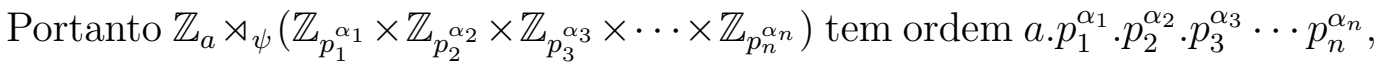
logo é um subgrupo normal de $\mathbb{Z}_{a} \rtimes_{\phi}\left(\mathbb{Z}_{p} \times \mathbb{Z}_{p_{1}^{\alpha_{1}}} \times \mathbb{Z}_{p_{2}^{\alpha_{2}}} \times \mathbb{Z}_{p_{3}^{\alpha_{3}}} \times \cdots \times \mathbb{Z}_{p_{n}^{\alpha_{n}}}\right)$ de índice $p$.

\section{Tipo II}

Seja $\mathbb{Z}_{a} \rtimes_{\phi}\left(\mathbb{Z}_{b} \times Q_{2^{t}}\right)$ um grupo do Tipo II da Tabela 3.1.

Considerando $b=p \cdot p_{1}^{\alpha_{1}} \cdot p_{2}^{\alpha_{2}} \cdot p_{3}^{\alpha_{3}} \cdots p_{n}^{\alpha_{n}}$, pelo mesmo raciocínio do Tipo I temos

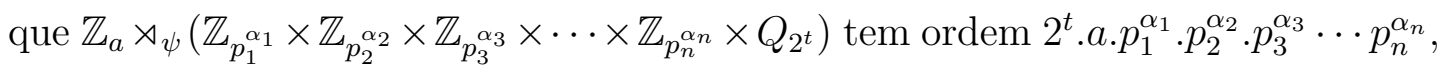
logo é um subgrupo normal de $\mathbb{Z}_{a} \rtimes_{\phi}\left(\mathbb{Z}_{p} \times \mathbb{Z}_{p_{1}^{\alpha_{1}}} \times \mathbb{Z}_{p_{2}^{\alpha_{2}}} \times \mathbb{Z}_{p_{3}^{\alpha_{3}}} \times \cdots \times \mathbb{Z}_{p_{n}^{\alpha_{n}}} \times Q_{2^{t}}\right)$ de índice $p$.

Observação 3.2.1 Concluimos que o método acima pode ser aplicado para todos os tipos da tabela 3.1. Temos então a tabela 3.2 de subgrupos normais onde $b_{p}=p_{1}^{\alpha_{1}} \cdot p_{2}^{\alpha_{2}} \cdot p_{3}^{\alpha_{3}} \cdots p_{n}^{\alpha_{n}}$ ou seja $b=p . b_{p}$, então $\mathbb{Z}_{b_{p}}=\mathbb{Z}_{p_{1}^{\alpha_{1}}} \times \mathbb{Z}_{p_{2}^{\alpha_{2}}} \times \mathbb{Z}_{p_{3}^{\alpha_{3}}} \times \cdots \times \mathbb{Z}_{p_{n}^{\alpha_{n}}}$.

Observação 3.2.2 Assim, dado um espaço de forma esférica $X=\Sigma^{2 n-1} / \gamma(H)$, onde $H$ é subgrupo da tabela 3.2. segue da Proposição 3.1.1 que $\pi_{1}\left(\Sigma^{2 n-1} / \gamma(H)\right) \simeq$ $H$ e portanto $\pi_{1}(X)$ não tem elementos de ordem $p$. 



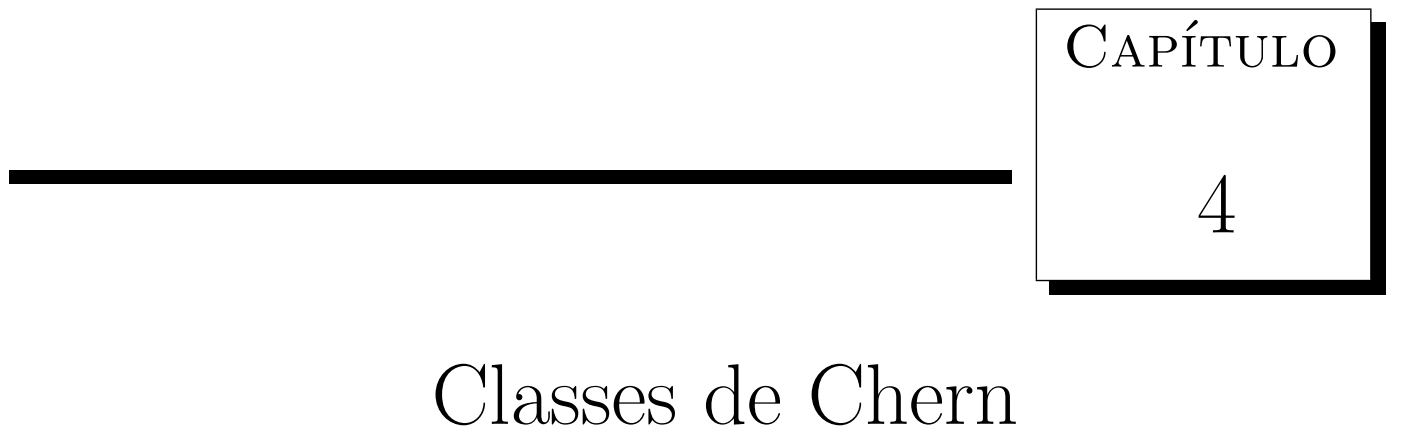

Neste capítulo apresentamos um estudo sobre as classes de Chern e sua relação com o conjunto de coincidências $A(f)$. Para maiores informações sobre classes de Chern ver [12]

\subsection{As Classes de Chern}

Definição 4.1.1 Dado um fibrado vetorial complexo $V$ sobre um espaço topológico X, a classe de Chern de Vé uma sequência de elementos da cohomologia de $X$ satisfazendo os seguintes axiomas:

1. $c_{0}(V)=1 \forall V$.

2. Se $f: Y \rightarrow X$ é contínua e $f^{*} V$ é o pullback do fibrado vetorial então $c_{k}\left(f^{*} V\right)=f^{*}\left(c_{k}(V)\right)$,

onde $c_{k}(V)$ é a k-ésima classe de Chern de $V$, que é um elemento de $H^{2 k}(X, \mathbb{Z})$.

3. Se $W \rightarrow X$ é outro fibrado vetorial complexo sobre $X$, então a classe de Chern da soma de Whitney $V \oplus W$ é dada por

$$
c(V \oplus W)=c(V) \cup c(W) \quad(\text { classe total })
$$


ou seja

$$
c_{k}(V \oplus W)=\sum_{i=0}^{k} c_{i}(V) \cup c_{k-i}(W)
$$

Observação 4.1.1 Pela definição das classes de Chern temos que

1. $c_{1}(V \oplus W)=c_{1}(V)+c_{1}(W)$.

2. $c_{p-1}(V \oplus W)=\sum_{i=0}^{p-1} c_{p-1-i}(V) \cup c_{i}(W)$.

3. $c_{i}(V \otimes W)=c_{i}(V)+c_{i}(W)$.

Agora, considere a seguinte formulação geral do Teorema de Borsuk - Ulam :

Definição 4.1.2 Sejam $X, Y$ dois espaços topológicos, $G$ um grupo finito agindo livremente em $X$ e $f: X \rightarrow Y$ uma aplicação contínua. Dizemos que um ponto $x \in X$ é um ponto de $G$ - coincidência de $f$ se a aplicação $f$ leva cada órbita $G x$ em um único ponto. O conjunto de todos os pontos de $G$ - coincidência de $f$ é denotado de $A(f)$. Dizemos que o Teorema de Borsuk-Ulam é válido para a terna $(X, G, Y)$ se $A(f) \neq \emptyset$, para toda função contínua $f: X \rightarrow Y$.

A técnica apresentada na sequência mostra que a validade do Teorema de Borsuk-Ulam para a terna $\left(M, G, \mathbb{R}^{m}\right)$, onde $M$ é uma variedade topológica, é equivalente a analisar zeros de seções contínuas em fibrados vetoriais especiais.

Definição 4.1.3 Seja $G$ um grupo e seja $R$ um anel comutativo com unidade. Então $I_{R}(G)$ denota o ideal aumentação do grupo anel $R[G]$, isto é, o núcleo do homomorfismo aumentação $I_{R}(G)=\operatorname{kernel}(\arg )$.

$$
\begin{gathered}
\arg : R[G] \rightarrow R \\
a_{1} g_{1}+a_{2} g_{2}+\cdots+a_{n} g_{n} \rightarrow a_{1}+a_{2}+\cdots+a_{n}
\end{gathered}
$$


Lema 4.1.1 Sejam $M$ uma variedade topológica e G um grupo finito agindo livremente em $M$. Dada uma aplicação $f: M \rightarrow \mathbb{R}^{m}$, definimos o conjunto de coincidências

$$
A(f)=\{x \in X \mid f(x)=f(g x) \forall g \in G\}
$$

Então toda seção contínua do fibrado vetorial $\xi_{M}: M \times_{G} I_{\mathbb{R}^{m}}(G) \rightarrow M / G$ possui um zero se, e somente se, $A(f) \neq \emptyset$

Demonstração. Temos que toda aplicação contínua $f: M \rightarrow \mathbb{R}^{m}$ dá origem a uma seção $\hat{s}(f): M / G \rightarrow M \times_{G} \mathbb{R}^{m}[G]$ do fibrado vetorial $\hat{\xi}: M \times_{G} \mathbb{R}^{m}[G] \rightarrow M / G$ definida pela fórmula

$$
\hat{s}(f)(x G)=\left(x, \sum_{g \in G} f\left(x g^{-1}\right) g\right) G
$$

Observe que $\hat{\xi}=\xi_{M} \oplus \varepsilon_{\mathbb{R}}^{m}$ onde $\varepsilon_{\mathbb{R}}^{m}$ é um fibrado vetorial real $m$-dimensional trivial. Assim a projeção $\pi: M \times{ }_{G} \mathbb{R}^{m}[G] \rightarrow M \times \times_{G} I_{\mathbb{R}^{m}}(G)$ está bem definida.

Agora defina uma seção contínua $s(f): M / G \rightarrow M \times_{G} I_{\mathbb{R}^{m}}(G)$ de $\xi_{M}$ pela fórmula $s(f)=\pi \circ \hat{s}(f)$. Portanto $s(f)(x G)=0$ se, e somente se, $f$ leva toda a órbita de $x \in M$ em um único ponto.

Reciprocamente, dada uma seção contínua $s$ de $\xi_{M}$, ela define uma aplicação $G$-equivariante $\bar{s}: M \rightarrow M \times \mathbb{R}^{m}[G]$ que, pela sua equivariância deve ser da forma

$$
\bar{s}(x)=\left(x, \sum_{g \in G} f\left(x g^{-1}\right) g\right)
$$

para $f: M \rightarrow \mathbb{R}^{m}$, e o lema segue.

Em virtude do importante Teorema 1.10.3, vamos analisar a classe de Euler e, consequentemente, a classe de Chern de determinados fibrados vetoriais.

Considere o fibrado vetorial complexo $\eta: E \mathbb{Z}_{p} \times_{\mathbb{Z}_{p}} I_{\mathbb{C}}\left(\mathbb{Z}_{p}\right) \rightarrow B \mathbb{Z}_{p}$. Vamos calcular a classe de Euler $(\bmod p)$ de $\eta$, a qual coincide com a sua classe de Chern $c_{p-1}(\eta)$. 
Temos que

$$
H^{*}\left(B \mathbb{Z}_{p}, \mathbb{Z}_{p}\right)=\Lambda_{\mathbb{Z}_{p}}(y) \otimes \mathbb{Z}_{p}[x]
$$

onde $\Lambda_{\mathbb{Z}_{p}}(y)$ é uma álgebra exterior com gerador 1-dimensional e $\mathbb{Z}_{p}[x]$ é uma álgebra polinomial com gerador 2-dimensional.

Lema 4.1.2 $e(\eta)=(-1) x^{p-1}$

Demonstração. A ação de $\mathbb{Z}_{p}$ em $\mathbb{C}$ pela rotação de $\frac{2 \pi}{p}$ induz em $\mathbb{C}$ um $\mathbb{C}\left[\mathbb{Z}_{p}\right]$ módulo que vamos denotar por L.

Seja $i: B \mathbb{Z}_{p} \rightarrow B \mathbb{Z}_{p}$ a identidade. Considere $\lambda$ o fibrado vetorial complexo 1-dimensional obtido do seguinte diagrama.

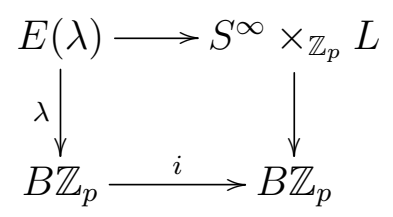

Temos que $c_{1}(\lambda)=x$.

Considere $\eta^{\prime}$ o fibrado vetorial obtido do seguinte diagrama

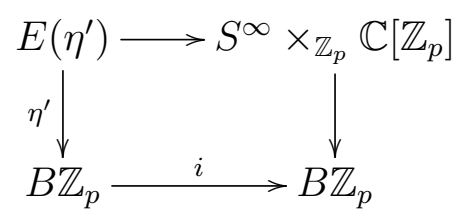

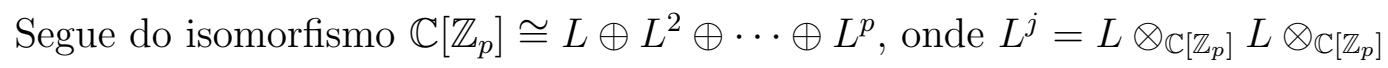
$\cdots \otimes_{\mathbb{C}\left[\mathbb{Z}_{p}\right]} L(j$-vezes $)$, que $\eta^{\prime} \cong \lambda \oplus \lambda^{2} \oplus \cdots \oplus \lambda^{p}$, onde $\lambda^{j}=\lambda \otimes_{\mathbb{C}} \lambda \otimes_{\mathbb{C}} \cdots \otimes_{\mathbb{C}} \lambda$ (j-vezes).

Observe que $\lambda^{p}$ é um fibrado complexo 1-dimensional trivial.

Temos que $\eta \oplus \varepsilon_{\mathbb{C}}^{1}=\eta^{\prime}$ onde $\varepsilon_{\mathbb{C}}^{1}$ é um fibrado vetorial complexo 1-dimensional trivial. 
Logo temos

$$
\eta \oplus \varepsilon_{\mathbb{C}}^{1} \cong \lambda \oplus \cdots \oplus \lambda^{p} \cong \lambda \oplus(\lambda \otimes \lambda) \oplus(\lambda \otimes \lambda \otimes \lambda) \oplus \cdots \oplus(\lambda \otimes \cdots \otimes \lambda)
$$

Pela Observação 4.1.1 temos que $c_{k}\left(\lambda^{j}\right)=0, \forall k \geq 2$ e $c_{1}\left(\lambda^{j}\right)=j c_{1}(\lambda)$.

Considere agora $\mu^{j}=\lambda^{j} \oplus \lambda^{j+1} \oplus \cdots \oplus \lambda^{p}$.

Assim,

$$
\begin{aligned}
c_{r}\left(\mu^{j}\right) & =c_{r}\left(\lambda^{j} \oplus \mu^{j+1}\right) \\
& =\sum_{i=1}^{r} c_{r-i}\left(\lambda^{j}\right) \cup c_{i}\left(\mu^{j+1}\right) \\
& =0+0+0+\cdots+0+c_{1}\left(\lambda^{j}\right) \cup c_{r-1}\left(\mu^{j+1}\right)+c_{0}\left(\lambda^{j}\right) \cup c_{r}\left(\mu^{j+1}\right) \\
& =c_{1}\left(\lambda^{j}\right) \cup c_{r-1}\left(\mu^{j+1}\right)+c_{r}\left(\mu^{j+1}\right)
\end{aligned}
$$

Em particular, $c_{p-k}\left(\mu^{j}\right)=c_{1}\left(\lambda^{j}\right) \cup c_{p-k-1}\left(\mu^{j+1}\right)+c_{p-k}\left(\mu^{j+1}\right)$ e observe que $c_{p-k}\left(\mu^{j}\right)=0, \forall j \geq k$.

De fato, primeiramente temos que $c_{p-k}\left(\mu^{p}\right)=c_{p-k}\left(\lambda^{p}\right)=0$ pois $p-k>1$.

Temos ainda $c_{p-k-(p-j)}\left(\mu^{j+(p-j)}\right)=c_{j-k}\left(\mu^{p}\right)=\left\{\begin{array}{l}0(\bmod p) j-k=1 ; \\ c_{j-k}\left(\lambda^{p}\right)=0 \quad j-k \geq 2 .\end{array}\right.$

Logo

$$
\begin{aligned}
c_{p-1}\left(\mu^{1}\right) & =c_{1}\left(\lambda^{1}\right) \cup c_{p-2}\left(\mu^{2}\right)+c_{p-1}\left(\mu^{2}\right) \\
& =c_{1}\left(\lambda^{1}\right) \cup\left(c_{1}\left(\lambda^{2}\right) \cup c_{p-3}\left(\mu^{3}\right)+c_{p-2}\left(\mu^{3}\right)\right) \\
& =c_{1}\left(\lambda^{1}\right) \cup\left(c_{1}\left(\lambda^{2}\right) \cup\left(c_{1}\left(\lambda^{3}\right) \cup c_{p-4}\left(\mu^{4}\right)+c_{p-3}\left(\mu^{4}\right)\right)\right) \\
& \cdot \\
& \cdot \\
& =c_{1}\left(\lambda^{1}\right) \cup c_{1}\left(\lambda^{2}\right) \cup c_{1}\left(\lambda^{3}\right) \cup \cdots \cup c_{1}\left(\lambda^{p-2}\right) \cup c_{1}\left(\lambda^{p-1}\right) .
\end{aligned}
$$

Agora como $c_{1}(\lambda)=x$ então $c_{p-1}\left(\mu^{1}\right)=x .2 x .3 x \cdots(p-2) x \cdot(p-1) x=(p-1)$ ! $x^{p-1}$.

O teorema de Wilson afirma que $(p-1) !=-1(\bmod \mathrm{p})$. Assim a classe de 
Euler $(\bmod p)$ do fibrado $\eta$ é $e(\eta)=c_{p-1}(\eta)=c_{p-1}\left(\eta \oplus \varepsilon_{\mathbb{C}}^{1}\right)=c_{p-1}\left(\mu^{1}\right)=(-1) x^{p-1}$.

Observação 4.1.2 Pela propriedade da universalidade existe o seguinte diagrama comutativo

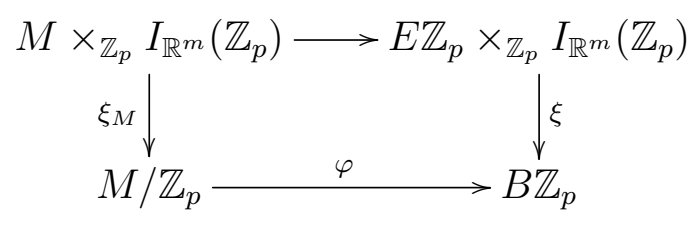

Temos que $\eta: E \mathbb{Z}_{p} \times_{\mathbb{Z}_{p}} I_{\mathbb{C}}\left(\mathbb{Z}_{p}\right) \rightarrow B \mathbb{Z}_{p}$, logo do isomorfismo $I_{\mathbb{C}^{m}} \cong I_{\mathbb{C}} \oplus I_{\mathbb{C}} \oplus$ $\cdots \oplus I_{\mathbb{C}} \cong m I_{\mathbb{C}}$, segue que $\mathbb{C} \xi \cong \eta \oplus \eta \oplus \cdots \oplus \eta \cong m \eta$ onde $\mathbb{C} \xi$ é a complexificação de fibrado $\xi$.

Assim $e(\xi)^{2}=e(\mathbb{C} \xi)=c_{1}(\mathbb{C} \xi)=c_{1}(\eta)^{m}=e(\eta)^{m}$. Pelo Lema 4.1.2 $e(\eta)=$ $(-1) x^{p-1}$ logo $e(\mathbb{C} \xi)=(-1)^{m} x^{m(p-1)}$. Portanto

$$
e(\xi)=a x^{\frac{m(p-1)}{2}}
$$

onde $a^{2} \equiv(-1)^{m}(\bmod p)$

Observação 4.1.3 $e\left(\xi_{M}\right)=\varphi^{*}(e(\xi))$. 


\section{Capítulo \\ 5 \\ O Teorema de Borsuk-Ulam para formas espaciais esféricas}

Neste capítulo apresentamos a demonstração do resultado principal derivado deste trabalho.

\subsection{Teorema Principal}

Teorema 5.1.1 Dada uma forma espacial esférica homotópica (2n-1)-dimensional $X$ com uma ação livre de $\mathbb{Z}_{p}, p>2$ primo, e uma aplicação contínua $f: X \rightarrow \mathbb{R}^{m}$ com $2 n-1 \geq m(p-1)$, então conjunto de coincidências $A(f)$ é não vazio. Mais ainda, $\operatorname{dim} A(f) \geq \operatorname{dim} X-m(p-1)$.

Demonstração. Considere $X$ um espaço de forma esférica homotópica, ou seja $X$ é da forma $X=\Sigma^{2 n-1} / \eta(H)$ onde $H$ é um grupo periódico finito. Como $X$ admite uma ação livre de $\mathbb{Z}_{p}$, então pelo Teorema 3.1.1 $H$ é um subgrupo de índice $p$ de um grupo finito e periódico $G$. Além disso $X / \mathbb{Z}_{p} \simeq\left(\Sigma^{2 n-1} / \eta(H)\right) / \mathbb{Z}_{p} \simeq \Sigma^{2 n-1} / \gamma(G)$.

Assim pelo Corolário 2.1 .1 temos $H^{l}\left(X / \mathbb{Z}_{p} ; \mathbb{Z}_{p}\right) \simeq H^{l}\left(\Sigma^{2 n-1} / \gamma(G) ; \mathbb{Z}_{p}\right) \stackrel{\psi}{\simeq}$ $H^{l}\left(G ; \mathbb{Z}_{p}\right)$ para $l \leq 2 n-1$. 
Logo da Observação 4.1 .3

$$
e\left(\xi_{X}\right) \in H^{m(p-1)}\left(X / \mathbb{Z}_{p} ; \mathbb{Z}_{p}\right) \simeq H^{m(p-1)}\left(\Sigma^{2 n-1} / \gamma(G) ; \mathbb{Z}_{p}\right)
$$

Pelo Teorema 2.3 .2 dado $b^{i} \in H^{2 i}\left(G ; \mathbb{Z}_{p}\right)$ com $p-1=i . s$ então $\left(b^{i}\right)^{\frac{m . s}{2}} \in$ $H^{i m s}\left(G ; \mathbb{Z}_{p}\right) \stackrel{\mathcal{\sim}}{\simeq} H^{m(p-1)}\left(\Sigma^{2 n-1} / \gamma(G) ; \mathbb{Z}_{p}\right) \simeq H^{m(p-1)}\left(X / \mathbb{Z}_{p} ; \mathbb{Z}_{p}\right)$ e $\psi\left(\left(b^{i}\right)^{\frac{m . s}{2}}\right)=$ $e\left(\xi_{X}\right)$.

Se $2 n-1 \geq m(p-1)$ então $\left(b^{i}\right)^{\frac{m . s}{2}} \neq 0$ o que implica $e\left(\xi_{X}\right) \neq 0$. Assim do Teorema 1.10 .3 toda seção contínua de $\xi_{X}$ possui um zero e, consequentemente pelo Lema 4.1.1 $A(f) \neq \emptyset$.

Agora vamos mostrar que $\operatorname{dim} A(f) \geq \operatorname{dim} X-m(p-1)$.

Como $A(f)$ é fechado e $G$-invariante, o conjunto $X \backslash A(f)$ também é $G$ invariante e assim podemos considerar a sequência exata do par

$$
\cdots \longrightarrow H^{n}\left(X / \mathbb{Z}_{p},(X \backslash A(f)) / \mathbb{Z}_{p}\right) \stackrel{\alpha}{\longrightarrow} H^{n}\left(X / \mathbb{Z}_{p}\right) \stackrel{\beta}{\longrightarrow} H^{n}\left(X \backslash A(f), \mathbb{Z}_{p}\right) \longrightarrow \cdots
$$

Considere $f^{\prime}: X \backslash A(f) \rightarrow \mathbb{R}^{m}$ e $\left(\xi_{X}\right)^{\prime}: X \backslash A(f) \times_{\mathbb{Z}_{p}} \mathbb{R}^{m}\left(\mathbb{Z}_{p}\right) \rightarrow(X \backslash A(f)) / \mathbb{Z}_{p}$. Como $A\left(f^{\prime}\right)=\emptyset$, pelo Lema 4.1.1 temos que $\left(\xi_{X}\right)^{\prime}$ possui uma seção não nula sobre $X \backslash A(f)$.

Assim $\beta\left(e_{p}\left(\xi_{X}\right)\right)=e_{p}\left(\left(\xi_{X}\right)^{\prime}\right)=0$. Segue da exatidão da sequência que existe um elemento não trivial

$$
\mu \in H^{m(p-1)}\left(X / \mathbb{Z}_{p},(X \backslash A(f)) / \mathbb{Z}_{p}\right)
$$

tal que $\alpha(\mu)=e_{p}\left(\xi_{X}\right)$.

Como estamos trabalhando com coeficientes no corpo $\mathbb{Z}_{p}$, existe um elemento não trivial correspondente

$$
\tilde{\mu} \in H_{m(p-1)}\left(X / \mathbb{Z}_{p},(X \backslash A(f)) / \mathbb{Z}_{p}\right) .
$$

Pela dualidade de Alexander, temos $H^{\operatorname{dim} X-m(p-1)}\left(A(f) / \mathbb{Z}_{p} ; \mathbb{Z}_{p}\right) \neq 0$ e assim 
$\operatorname{dim}_{\mathbb{Z}_{p}} A(f) / \mathbb{Z}_{p} \geq \operatorname{dim} X-m(p-1)$.

Como $\mathbb{Z}_{p}$ é um grupo finito segue que $\operatorname{dim} A(f) \geq \operatorname{dim} A(f) / \mathbb{Z}_{p} \geq \operatorname{dim} X-$ $m(p-1)$.

Observação 5.1.1 Note que, se o período de $G$ é $k$ então $k-1 \geq$ m.i.s. 



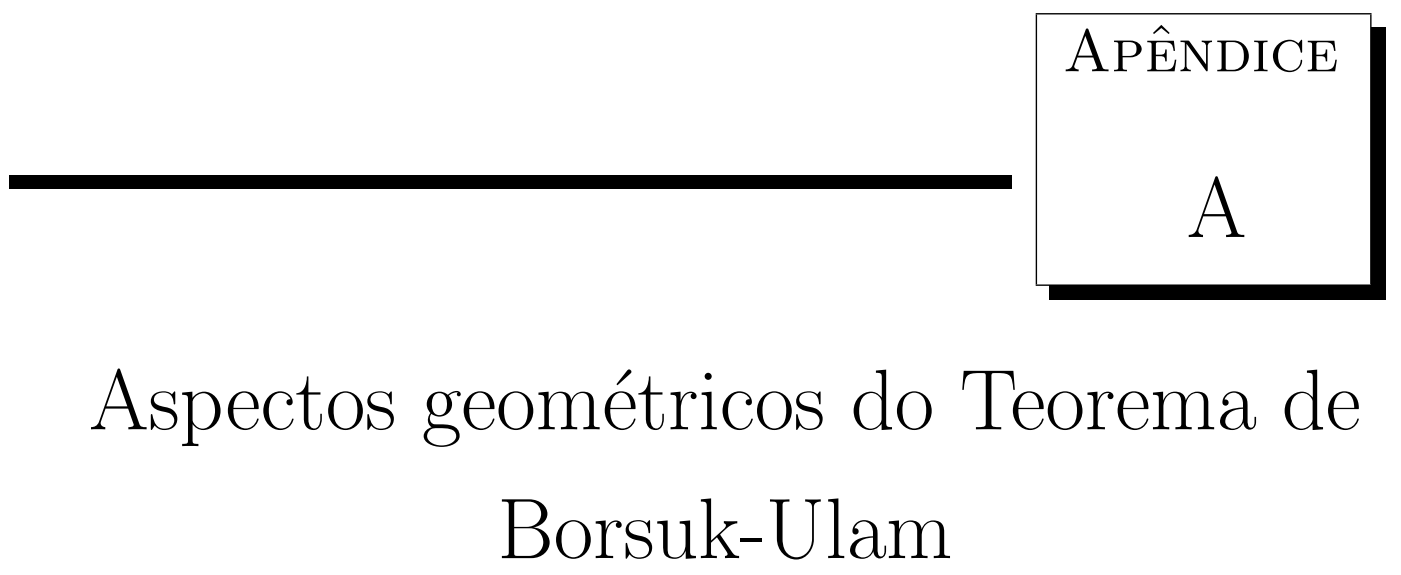

Neste capítulo apresentamos alguns aspectos geométricos do Teorema de Borsuk-Ulam para o caso de ações livres do grupo cíclico $\mathbb{Z}_{p}$

A.1 O teorema de Borsuk-Ulam para $\mathbb{Z}_{p}$-ações livres

O teorema clássico de Borsuk-Ulam possui as seguintes versões equivalentes bem conhecidas:

1. Para toda função contínua $f: S^{n} \rightarrow \mathbb{R}^{n}$ existe um ponto $x \in S^{n}$ com $f(x)=f(-x)$.

2. Não existe aplicação equivariante $f: S^{n} \rightarrow S^{n-1}$ com relação a aplicação antipoda.

Definição A.1.1 Dizemos que a tripla $(X, \tau ; Y)$ satisfaz o Teorema de BorsukUlam para ações livres de $\mathbb{Z}_{p}$ se dada qualquer aplicação contínua $f: X \rightarrow Y$ o 
conjunto de coincidências $A(f)=\left\{x \in X \mid f(x)=f\left(\tau^{j}(x)\right) j=1, \cdots, p-1\right\}$ é não vazio.

No caso particular em que $p=2$, temos as seguintes versões equivalentes do Teorema de Borsuk-Ulam:

Proposição A.1.1 Seja $(X, \tau)$ um espaço com uma ação livre de $\mathbb{Z}_{2}$. Então são equivalentes:

1. O Teorema de Borsuk-Ulam para $\mathbb{Z}_{2}$-ações livres é valido para a tripla $\left(X, \tau ; \mathbb{R}^{n}\right)$

2. Não existe aplicação $\mathbb{Z}_{2}$-equivariante $f: X \rightarrow S^{n-1}$.

Demonstração. $(1 \Rightarrow 2)$. Se existe aplicação equivariante $f: X \rightarrow S^{n-1} \subset \mathbb{R}^{n}$, então $f(\tau(x))=-f(x) \neq f(x)$, para todo $x \in X$ e, portanto, o Teorema de Borsuk-Ulam não é válido para a tripla $\left(X, \tau ; \mathbb{R}^{n}\right)$.

$(2 \Rightarrow 1)$. Se o Teorema de Borsuk-Ulam não for válido para a tripla $\left(X, \tau ; \mathbb{R}^{n}\right)$, então está bem definida a aplicação equivariante $F: X \rightarrow S^{n-1}$, dada por

$$
F(x)=\frac{f(x)-f(\tau(x))}{\|f(x)-f(\tau(x))\|} .
$$

O objetivo nesta seção é mostrar uma condição equivalente ao Teorema de Borsuk-Ulam para a tripla $\left(X, \tau ; \mathbb{R}^{n}\right)$, onde $\tau$ determina uma ação livre de $\mathbb{Z}_{p}$ sobre $X$, a qual estende a Proposição A.1.1. Observamos que uma tentativa direta de estender a demonstração da Proposição A.1.1 para o caso de $\mathbb{Z}_{p}$-ações livres, $p>2$ primo, não é possível pois os argumentos utilizados na prova dependem do fato de $p$ ser igual a 2. A seguir, apresentamos uma técnica a qual permite a obtenção do resultado pretendido para o caso de $\mathbb{Z}_{p}$-ações livres, $p>2$ primo.

$$
\begin{aligned}
& \text { Considere }\left(\mathbb{R}^{n}\right)_{\Delta}^{p}=\prod_{i=1}^{p}\left(\mathbb{R}^{n}\right)^{i}-\Delta, \text { onde } \\
& \quad \Delta=\left\{\left(x_{1}, x_{2}, \cdots, x_{p}\right) \in \prod_{i=1}^{p}\left(\mathbb{R}^{n}\right)^{i} ; x_{1}=x_{2}=\cdots=x_{p}\right\}
\end{aligned}
$$


é a diagonal usual em $\prod_{i=1}^{p}\left(\mathbb{R}^{n}\right)^{i}$. Então, $\left(\mathbb{R}^{n}\right)_{\Delta}^{p}$ admite uma ação livre de $\mathbb{Z}_{p}$, gerada por um homeomorfismo periódico $\sigma:\left(\mathbb{R}^{n}\right)_{\Delta}^{p} \rightarrow\left(\mathbb{R}^{n}\right)_{\Delta}^{p}$ de período $p$ dado por

$$
\sigma\left(x_{1}, x_{2}, \cdots, x_{p}\right)=\left(x_{2}, x_{3}, \cdots, x_{p}, x_{1}\right)
$$

Lema A.1.1 Existe uma equivalência de homotopia $\mathbb{Z}_{p}$-equivariante

$$
g:\left(\left(\mathbb{R}^{n}\right)_{\Delta}^{p}, \sigma\right) \rightarrow\left(S^{n(p-1)-1}, \gamma\right)
$$

onde $\gamma$ é a ação padrão de $\mathbb{Z}_{p}$ sobre $S^{n(p-1)-1}$.

Demonstração. Vamos interpretar $\left(\mathbb{R}^{n}\right)^{p}$ como espaço das matrizes $\left(x_{i j}\right) i=$ $1, \cdots, n$ e $j=1, \cdots, p$ com $n$ linhas e $p$ colunas. Assim $\sigma$ é a permutação cíclica das colunas.

Os elementos de $\left(\mathbb{R}^{n}\right)_{\Delta}^{p}$ são todas as matrizes dessa forma exceto aquelas que tem todas as colunas iguais.

Vamos considerar $D$ o subespaço $n(p-1)$-dimensional que é perpendicular a diagonal e seja $g_{1}:\left(\mathbb{R}^{n}\right)^{p} \rightarrow D$ a projeção ortogonal de $\left(\mathbb{R}^{n}\right)^{p}$ em $D$. Em coordenadas $D$ é o subespaço consistindo de todas as matrizes $n \times p$ tal que as somas dos elementos de cada linha é zero, ou seja $\sum_{j=1}^{p} x_{i j}=0 \forall i$

$$
\left[\begin{array}{ccccc}
x_{11} & x_{12} & \cdot & \cdot & x_{1 p} \\
x_{21} & x_{22} & \cdot & \cdot & x_{2 p} \\
\cdot & \cdot & \cdot & \cdot & \cdot \\
\cdot & \cdot & \cdot & \cdot & \cdot \\
x_{n 1} & x_{n 2} & \cdot & \cdot & x_{n p}
\end{array}\right] .
$$

De fato, se $X \in D$ e $Y \in \Delta$, então $X . Y^{t}=0$. 


$$
X=\left[\begin{array}{ccccc}
x_{11} & x_{12} & \cdot & \cdot & x_{1 p} \\
x_{21} & x_{22} & \cdot & \cdot & x_{2 p} \\
\cdot & \cdot & \cdot & \cdot & \cdot \\
\cdot & \cdot & \cdot & \cdot & \cdot \\
x_{d 1} & x_{d 2} & \cdot & \cdot & x_{n p}
\end{array}\right] \text { e } Y=\left[\begin{array}{ccccc}
y_{1} & y_{1} & \cdot & \cdot & y_{1} \\
y_{2} & y_{2} & \cdot & \cdot & y_{2} \\
\cdot & \cdot & \cdot & \cdot & \cdot \\
\cdot & \cdot & \cdot & \cdot & \cdot \\
y_{n} & y_{n} & \cdot & \cdot & y_{n}
\end{array}\right]
$$

Como $X . Y^{t}=0$ então

$$
\left\{\begin{array} { c } 
{ y _ { 1 } ( x _ { 1 1 } + x _ { 1 2 } + \cdots + x _ { 1 p } ) = 0 } \\
{ y _ { 2 } ( x _ { 2 1 } + x _ { 2 2 } + \cdots + x _ { 2 p } ) = 0 } \\
{ \cdot } \\
{ y _ { d } ( x _ { n 1 } + x _ { n 2 } + \cdots + x _ { n p } ) = 0 }
\end{array} \Longrightarrow \left\{\begin{array}{c}
x_{11}+x_{12}+\cdots+x_{1 p}=0 \\
x_{21}+x_{22}+\cdots+x_{2 p}=0 \\
\cdot \\
\cdot \\
x_{n 1}+x_{n 2}+\cdots+x_{n p}=0
\end{array}\right.\right.
$$

Assim $g_{1}$ leva a matriz $X=\left(x_{i j}\right)$ na matriz $g_{1}(X)=\left(x_{i j}-\frac{1}{p} \sum_{k=1}^{p} x_{i k}\right)_{i j}$. Ou seja a média das colunas é retirada de cada coluna.

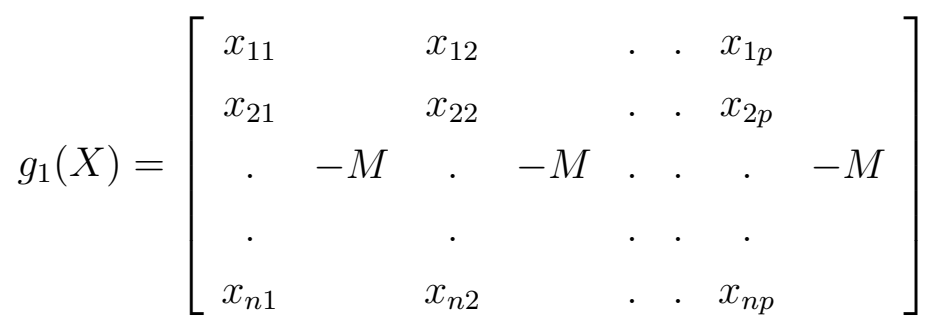

onde $M=\frac{1}{p}\left[\begin{array}{c}x_{11}+x_{12}+\cdots+x_{1 p} \\ x_{21}+x_{22}+\cdots+x_{2 p} \\ \cdot \\ \cdot \\ x_{n 1}+x_{n 2}+\cdots+x_{n p}\end{array}\right]$

Temos que $g_{1}(X)$ é a matriz nula se, e somente se, cada coluna de X é igual a média das colunas $\mathrm{M}$, ou seja todas as colunas são iguais. Logo $g_{1}:\left(\mathbb{R}^{n}\right)_{\Delta}^{p} \rightarrow$ $D-\{0\}$ é sobrejetora. 
Seja $g:\left(\mathbb{R}^{n}\right)_{\Delta}^{p} \rightarrow S(D), g(X)=\frac{g_{1}(X)}{\left\|g_{1}(X)\right\|}$ onde $(S(D), \tau)$ é a esfera unitária em $D$ e $\tau=\left.\sigma\right|_{S(D)}$ é a restrição de $\sigma$ a $S(D)$, a qual pode ser identificada equivariantemente com $\left(S^{n(p-1)-1}, \gamma\right)$ e, neste caso a inversa homotópica de $g$ é a aplicação inclusão $i: S^{n(p-1)-1}=S(D) \rightarrow\left(\mathbb{R}^{n}\right)_{\Delta}^{p}$, a qual é claramente equivariante.

Para que $g$ seja $\mathbb{Z}_{p}$-equivariante mostremos que $g \circ \sigma=\tau \circ g$. Temos

$$
\begin{aligned}
& X=\left[\begin{array}{ccccc}
x_{11} & x_{12} & \cdot & . & x_{1 p} \\
x_{21} & x_{22} & \cdot & \cdot & x_{2 p} \\
\cdot & \cdot & \cdot & \cdot & \cdot \\
\cdot & \cdot & \cdot & \cdot & \cdot \\
x_{n 1} & x_{n 2} & . & . & x_{n p}
\end{array}\right], \sigma(X)=\left[\begin{array}{cccccc}
x_{12} & x_{13} & \cdot & \cdot & x_{1 p} & x_{11} \\
x_{22} & x_{23} & \cdot & \cdot & x_{2 p} & x_{21} \\
\cdot & \cdot & \cdot & \cdot & \cdot & \cdot \\
\cdot & \cdot & \cdot & \cdot & \cdot & \cdot \\
x_{n 2} & x_{n 3} & \cdot & \cdot & x_{n p} & x_{n 1}
\end{array}\right]
\end{aligned}
$$

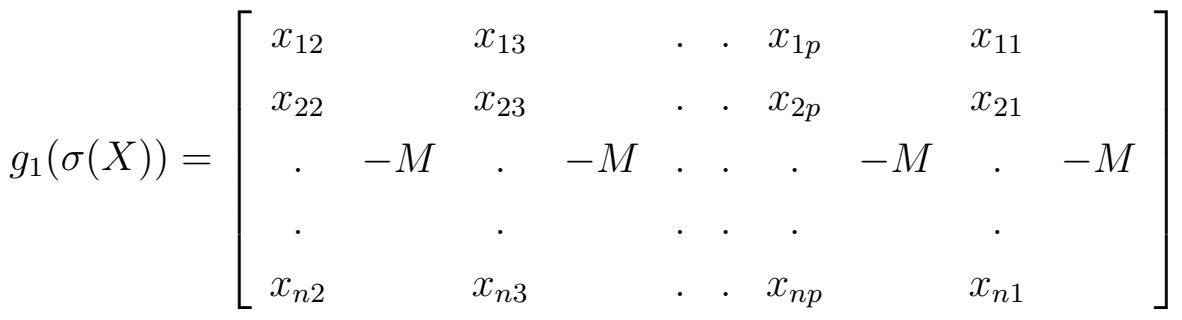

$\operatorname{Logo} \sigma\left(g_{1}(X)\right)=g_{1}(\sigma(X))$ e $\left\|g_{1}(X)\right\|=\left\|g_{1}(\sigma(X))\right\|=\left\|\sigma\left(g_{1}(X)\right)\right\|$ e segue que

$$
\begin{aligned}
g(\sigma(X)) & =\frac{g_{1}(\sigma(X))}{\left\|g_{1}(\sigma(X))\right\|} \\
& =\frac{\sigma\left(g_{1}(X)\right)}{\left\|g_{1}(X)\right\|} \\
& =\left.\sigma\right|_{S(D)}\left(\frac{g_{1}(X)}{\left\|g_{1}(X)\right\|}\right) \\
& =\left.\sigma\right|_{S(D)}(g(X)) .
\end{aligned}
$$




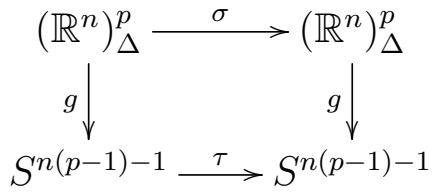

Agora, vamos mostrar uma condição equivalente ao Teorema de Borsuk-Ulam para ações livres de $\mathbb{Z}_{p}$, a qual estende a Proposição A.1.1.

Proposição A.1.2 Seja $(X, \tau)$ um espaço com uma ação livre de $\mathbb{Z}_{p}$. Então são equivalentes:

1. O Teorema de Borsuk-Ulam para $\mathbb{Z}_{p}$-ações livres é valido para a tripla $\left(X, \tau ; \mathbb{R}^{n}\right)$;

2. Não existe aplicação $\mathbb{Z}_{p}$-equivariante $F:(X, \tau) \rightarrow\left(S^{n(p-1)-1}, \gamma\right)$, onde $\gamma$ é a ação padrão de $\mathbb{Z}_{p}$ sobre $S^{n(p-1)-1}$.

Demonstração. $(1 \Rightarrow 2)$. Considere a inclusão $i:\left(S^{n(p-1)-1}, \gamma\right) \rightarrow\left(\left(\mathbb{R}^{n}\right)_{\Delta}^{p}, \sigma\right)$, a qual pelo Lema A.1.1é a inversa homotópica equivariante da aplicação $g$. Suponha que exista uma aplicação $\mathbb{Z}_{p}$-equivariante

$$
F:(X, \tau) \rightarrow\left(S^{n(p-1)-1}, \gamma\right) \subset\left(\left(\mathbb{R}^{n}\right)_{\Delta}^{p}, \sigma\right)
$$

e sejam $f_{i}: X \rightarrow \mathbb{R}^{n}$ dadas por $f_{i}=p_{i} \circ F$, onde $p_{i}$ é a projeção na i-ésima coordenada $i=1, \ldots, p$.

Temos

$$
F\left(\tau^{j} x\right)=\sigma^{j} F(x), \forall x \in X, \forall j=1, \ldots, p-1
$$

$\mathrm{e}$

$$
F(x)=\left(f_{1}(x), f_{2}(x) \ldots, f_{p}(x)\right)
$$


Assim

$$
\begin{aligned}
F\left(\tau^{j} x\right) & =\left(f_{1}\left(\tau^{j} x\right), \ldots, f_{p}\left(\tau^{j} x\right)\right) \\
\sigma^{j} F(x) & =\left(f_{j+1}(x), \ldots, f_{j-1}(x)\right)
\end{aligned}
$$

Logo, $f_{1}\left(\tau^{j} x\right)=f_{j+1}(x), \forall x \in X, \forall j=1, \ldots, p-1$ e como $F(x)=$ $\left(f_{1}(x), \ldots, f_{p}(x)\right) \in\left(\mathbb{R}^{n}\right)_{\Delta}^{p}$ concluímos que o Teorema de Borsuk-Ulam para $\mathbb{Z}_{p}$-ações livres não é valido para a tripla $\left(X, \tau ; \mathbb{R}^{n}\right)$, pois $f_{i}: X \rightarrow \mathbb{R}^{n}$ não possui $x \in X$ tal que $f(x)=f\left(\tau^{j}(x)\right), \forall j=1, \ldots, p-1$ caso contrario $\left(f_{1}(x), f_{2}(x) \ldots, f_{p}(x)\right) \in \Delta$.

$(2 \Rightarrow 1)$. Suponha que o Teorema de Borsuk-Ulam para $\mathbb{Z}_{p}$-ações livres não é válido para a tripla $\left(X, \tau ; \mathbb{R}^{n}\right)$. Então existe uma função contínua $f: X \rightarrow \mathbb{R}^{n}$, para a qual não existe um ponto $x \in X$ tal que $f(x)=f\left(\tau^{j}(x)\right), \forall j=1, \ldots, p-1$.

Assim, podemos definir a aplicação $G: X \rightarrow\left(\mathbb{R}^{n}\right)_{\Delta}^{p}$ dada por

$$
G(x)=\left(f(x), f(\tau x), \ldots, f\left(\tau^{p-1} x\right)\right)
$$

Temos que $G \circ \tau=\sigma \circ G, \log \sigma G$ é $\mathbb{Z}_{p}$-equivariante. Usando a aplicação equivariante $g$ dada pelo Lema A.1.1 temos que a composição

$$
F=g \circ G:(X, \tau) \rightarrow\left(S^{n(p-1)-1}, \gamma\right)
$$

é uma aplicação equivariante. 



\section{Referências Bibliográficas}

[1] ADEM, A.; MILGRAM, R.J. Cohomology of finite groups. SpringerVerlag, 1994.

[2] BIASI, C.; MATTOS, D.; SANTOS E.L. A Borsuk-Ulam Theorem for maps from a sphere to a generalized manifold. Geom. Dedicata v. 107, p. 101-110, 2004 .

[3] BORSUK, K. Drei Sätze über die $n$-dimensionale euklidische Sphäre, Fund. Math. v. 20, p. 177-190, 1933.

[4] BROWN, K. S. Cohomology of Groups. New York: Springer-Verlag, 1982.

[5] CONNER, P.; FLOYD, E. Differentiable periodic maps, Springer Verlag, Berlin, 1964.

[6] DAVIS, J. F.; KIRK, P. Lecture Notes in Algebraic Topology. v. 35, Graduate studies in mathematics, 2001.

[7] GONÇALVES D.L.; JAWOROWSKI J.; PERGHER P.L.Q. Measuring the size of the coincidence set, Topology Appl. v. 125, p. 465-470, 2002.

[8] GONÇALVES D.L.; JAWOROWSKI J.; PERGHER P.L.Q. G-coincidences for maps of homotopy spheres into CW-complexes, Proc. Amer. Math. Soc. v. 130, n. 10, p. 3111-3115, 2002. 
[9] GONÇALVES D.L.; JAWOROWSKI J.; PERGHER P.L.Q.; VOLOVIKOV A.Y. Coincidences for maps of spaces with finite group actions. Topology Appl. v145, n 1-3, p.61-68, 2004.

[10] GONÇALVES D.L.; PERGHER P.L.Q. $\mathbb{Z}_{p}$-coincidence for maps of spheres into CW-complexes, Kobe J. Math. v.15, p.191 - 195, 1998.

[11] GONÇALVES D.L.; MANZOLI NETO O.; SPREAFICO M. The BorsukUlam Theorem for homotopy spherical space forms. Journal of Fixed Point Theory and Applications v.9, p. 285-294, 2011.

[12] GROTHENDIECK A. La Théorie des Classes de Chern, Bull.Soc.math. France v.86, p.137-154, 1958.

[13] IZYDOREK M. On the Borsuk-Ulam Theorem for Free $\mathbb{Z}_{p}$-Action, Bulletin of the Polish Academy of Sciences Math. v.38, 1990. 15-21.

[14] JAWOROWSKI J. Periodic coincidence for maps of spheres, Kobe J. Math. v.17, p.21-26, 2000.

[15] LIMA, E.L. Grupos Fundamentais e Espaço de Recobrimento. Rio de Janeiro, Projeto Euclides, Impa, 2006.

[16] MAUTOUSEK J. Using the Borsuk-Ulam Theorem - Lectures on Topological Methods in Combinatorics and Geometry. Springer ,2003.

[17] PICCININI R.; SPREAFICO M. Conjugacy Classes of Gauge Groups. Queens Papers in Pure and Applied Math. v.111, 1998.

[18] SWAN, R. G. Periodic resolutions for finite groups. Annal of Mathematics v.72, p. 267-291, 1960.

[19] SANTOS M. D. V.; ANDRADE M. G. C. Cohomologia De Grupos Finitos e G-Coincidências De Aplicações, 2010, Dissertação de Mestrado, IBLICE-UNESP. 
[20] TURYGIN, Y.A. Borsuk-Ulam Property of Finite Group Actions on Manifolds and Applications. 2007, Tese de Doutorado, University of Florida.

[21] VOLOVIKOV A.Y. Coincidence points of mappings of $\mathbb{Z}_{p}^{n}$-spaces. Izv. Ross. Akad. Nauk Ser. Mat. v.69, no. 5, p.53-106, 2005.

[22] WOLF, J. A. Spaces of constant curvature. MacGraw-Hill, Inc., 1967. 



\section{Índice Remissivo}

$\mathbb{Z}$ G-módulos, 10,11

ação transitiva, 4

anel de cohomologia, 26

aplicação aumentação, 7

aplicação diagonal, 11

aplicação equivariante, 2

classes de Chern, 34, 36, 37

cohomologia periódica, 24

coinvariantes, 7

CW-complexo, 4, 5, 21, 24

espaço de forma esférica, 1, 39, 40

espaços de recobrimento, 24

fibra, 3, 22

forma espacial esférica, 1

grupo índice primo, 30

ideal aumentação, 34

invariantes, 7

$\mathrm{K}(\mathrm{G}, 1)$-complexo, 10

número de folhas do recobrimento, 4 número de Lefschetz, 22

operador bordo, 10

operador cobordo, 10

produto cup, 11

propriamente descontínuo, 4

recobrimento regular, 3, 4, 22

recobrimento universal, 3, 4

resolução livre, 6, 7

resoluções projetivas, 6

sequência espectral, 24

Teorema de Borsuk-Ulam, 39

vizinhança elementar, 3 